\title{
On Davis-Januszkiewicz homotopy types II: Completion and globalisation
}

\author{
DIETRICH NOTBOHM \\ NigEL RAY
}

\begin{abstract}
For any finite simplicial complex $K$, Davis and Januszkiewicz defined a family of homotopy equivalent $\mathrm{CW}$-complexes whose integral cohomology rings are isomorphic to the Stanley-Reisner algebra of $K$. Subsequently, Buchstaber and Panov gave an alternative construction, which they showed to be homotopy equivalent to the original examples. It is therefore natural to investigate the extent to which the homotopy type of a space $X$ is determined by such a cohomology ring. Having analysed this problem rationally in Part I, we here consider it prime by prime, and utilise Lannes' $T$-functor and Bousfield-Kan type obstruction theory to study the $p$-completion of $X$. We find the situation to be more subtle than for rationalisation, and confirm the uniqueness of the completion whenever $K$ is a join of skeleta of simplices. We apply our results to the global problem by appealing to Sullivan's arithmetic square, and deduce integral uniqueness whenever the Stanley-Reisner algebra is a complete intersection.
\end{abstract}

55P15, 55P60; 05E99

\section{Introduction}

For any finite simplicial complex $K$, the Stanley-Reisner algebra $\mathbb{Z}[K]$ is an important combinatorial invariant (see Stanley [14]), and may be graded by assigning dimension 2 to each of its generators. The corresponding $R$-algebra $R[K]$ is defined over any commutative ring $R$ as $R \otimes \mathbb{Z}[K]$, and a topological space $X$ realises $R[K]$ whenever $H^{*}(X ; R)$ is isomorphic to $R[K]$ as graded $R$-algebras. In their pioneering work on toric topology [5], Davis and Januszkiewicz construct a family of realisations of $\mathbb{Z}[K]$ for every $K$, and show each of them to be homotopy equivalent to a certain universal example. We refer to a generic representative of this homotopy type as a Davis-Januszkiewicz space $\mathrm{DJ}(K)$.

Questions of uniqueness then arise, and suggest that we investigate the relationship between the geometric properties of $K$ and the number of homotopy types which realise $\mathbb{Z}[K]$. As usual, the problem is best approached by dealing separately with 
its rational and $p$-adic versions, and applying Sullivan's arithmetic square to recover global information. We consider the rational situation in our paper [11], and study the case in which $\mathbb{Q}[K]$ is a complete intersection. The rational homotopy type of any realisation of $\mathbb{Q}[K]$ is then unique, up to weak equivalence of nilpotent spaces.

Our purpose here is to address the $p$-adic version of the problem. We obtain uniqueness statements for a somewhat larger class of complexes $K$, from which we deduce global uniqueness for complete intersections by appealing to the arithmetic square. So far, we have little evidence to suggest that these results may be extended to more general $K$, either $p$-adically or globally; nor, however, do we have any example of an exotic Davis-Januszkiewicz space that realises $\mathbb{Z}[K]$ yet fails to be homotopy equivalent to $\mathrm{DJ}(K)$. On the other hand, a simple cohomological argument proves that the suspension of any such space is equivalent to $\Sigma \mathrm{DJ}(K)$, for every $K$.

We refer readers to [11] for detailed background and prerequisites, and summarise the crucial points in Section 2 below, where we also state our main results explicitly. Unlike the rational version, we have found no helpful formulation of the $p$-adic problem in terms of model category theory. Nevertheless, we work in several algebraic and geometric categories which admit model structures, and appeal regularly to the language of homotopy colimits. We insist, for example, that our spaces lie in the model category TOP of $k$-spaces and continuous maps (see Vogt [16]). Sometimes we deal with based CW-complexes $(X, *)$, which lie in the pointed category $\mathrm{TOP}^{+}$; then $*$ is a distinguished $0-$ cell and its inclusion into $X$ is a cofibration.

So far as notation is concerned, we let $p$ denote a fixed but arbitrary prime throughout our work. We usually abbreviate the cyclic group $\mathbb{Z} / p$ to $C$ in the interests of notational simplicity, and write $\mathbb{Z}_{p}^{\wedge}$ for the $p$-adic integers. We denote the field of $p$ elements by $\mathbb{F}_{p}$ and the topological group of unimodular complex numbers by $T$, in order to distinguish them from the underlying group $\mathbb{Z} / p$ and the underlying circle $S^{1}$ respectively. Over any commutative $\operatorname{ring} R$ with identity, we interpret the polynomial algebra generated by a set $V$ of graded independent variables as the symmetric algebra $S_{R}(V)$ on $V$. Finally, for every pair of objects $x_{0}$ and $x_{1}$ of an arbitrary category $\mathrm{C}$, we write $\mathrm{C}\left(x_{0}, x_{1}\right)$ for the set of morphisms $x_{0} \rightarrow x_{1}$.

For any space $X$ we consider the $p$-adic completion $X_{p}^{\wedge}$ described by Bousfield and Kan [2], which agrees with Sullivan's original $p$-completion in all our cases. Given a $p$-complete space $X_{K}$ realising $\mathbb{Z}_{p}^{\wedge}[K]$, our underlying strategy is to develop methods of comparing $X_{K}$ with a canonical representative $\mathrm{hc}(K)$ for $\mathrm{DJ}(K)$, and each of Sections 3 to 8 takes steps towards this goal. We organise the programme as follows. In Section 3, we begin by assuming that $X_{K}$ realises $\mathbb{F}_{p}[K]$, and apply Lannes' $T$ functor to compute the mod $p$ cohomology ring of certain components of the mapping 
space $\operatorname{map}\left(B C^{\sigma}, X_{K}\right)$ for appropriate elementary abelian $p$-groups $C^{\sigma}$. We extend our computations to $p$-adic cohomology in Section 4 , by taking advantage of the fact that the mod $p$ ring is zero in odd dimensions. In Section 5 we develop the corresponding results for certain components of $\operatorname{map}\left(B T^{\sigma}, X_{K}\right)$, by interpreting $T_{p}^{\wedge}$ as a limit of cyclic subgroups $\mathbb{Z} / p^{r}$.

In order to bring these calculations to bear on the central problem, we provide a brief survey of higher limits of algebraic $\operatorname{CAT}(K)$-diagrams in Section 6. These feature prominently in the obstruction theory associated to Bousfield and Kan's spectral sequence for homotopy limits, which we investigate in Section 7; in particular, we show that there are no obstructions to defining a homotopy equivalence $\operatorname{hc}(K)_{p}^{\wedge} \rightarrow X_{K}$ when $K$ is the skeleton of a simplex, and deduce $p$-adic uniqueness in such cases. We extend the results to iterated joins in Section 8 , where we also confirm that homotopy classes of self-equivalences of $X_{K}$ are classified by their action on cohomology. Finally, in Section 9, we employ the arithmetic square to combine our $p$-adic conclusions with the rational calculations of [11], and prove global uniqueness for $X_{K}$ when $K$ is a complete intersection. The latter is equivalent to identifying $K$ as the iterated join of a simplex with boundaries of simplices.

The authors would like to reiterate their gratitude to the organisers of the International Conference on Algebraic Topology, held on the Island of Skye in June 2001, for providing the environment in which their collaboration began; and to the London Mathematical Society for its support of the Transpennine Topology Triangle, whose meetings have ensured that it continues to flourish. They are also indebted to their colleagues Tony Bahri, who encouraged us to include our remarks on the suspension of $\mathrm{DJ}(K)$, and Taras Panov, who contributed so generously to many of our discussions.

\section{Preliminaries and main results}

We shall work with a canonical representative for $\mathrm{DJ}(K)$, constructed as the homotopy colimit of a diagram of topological spaces. Our methods depend upon the cohomological and homotopy theoretic properties of such diagrams, so we recall the crucial definitions and notation in this section. Readers may consult [11] for further motivation and details.

We consider an ordered set $V$ of vertices $v_{1}, \ldots, v_{m}$. A simplicial complex $K$ on $V=V(K)$ is given by a finite set of faces $\sigma \subseteq V$, which is closed with respect to the formation of subsets. The dimension $\operatorname{dim}(\sigma)$ of any face is given in terms of its cardinality by $|\sigma|-1$, and $\operatorname{dim} K$ is the maximum dimension attained by its faces. We assume that the empty face $\varnothing$ belongs to $K$ unless otherwise stated, and write $K^{\times}$when we wish to emphasise that it has been omitted. The most basic 
example is the simplex $\Delta(V)$; it consists of all possible subsets of $V$, and therefore contains any $K$ on $V$ as a subcomplex. Its $r$-skeleton $\Delta^{(r)}(V)$ includes those faces $\sigma \subset V$ of dimension $\leq r$, for any $0 \leq r \leq m-1$. In particular, $\Delta^{(m-2)}(V)$ is the boundary $\partial(V) \subset \Delta(V)$, constructed by deleting the maximal face $V$. For any two disjoint simplicial complexes $K$ and $L$, their join $K * L$ on $V(K) \cup V(L)$ has faces $\{\sigma \cup \tau: \sigma \in K, \tau \in L\}$; thus $K$ and $L$ are full subcomplexes.

Every face $\sigma \in K$ determines several subcomplexes. In particular, we need the simplex $\Delta(\sigma)$ on $\sigma$, and the link and star of $\sigma$, defined by

$$
\ell_{K}(\sigma):=\{\tau \backslash \sigma: \sigma \subseteq \tau \in K\} \quad \text { and } \quad \operatorname{st}_{K}(\sigma):=\{\tau: \sigma \cup \tau \in K\} ;
$$

so $\operatorname{st}_{K}(\sigma)$ coincides with $\Delta(\sigma) * \ell_{K}(\sigma)$ as subcomplexes of $K$. Given any two complexes $K$ and $L$, we note that

$$
\ell_{K * L}(\sigma \cup \tau)=\ell_{K}(\sigma) * \ell_{L}(\tau) \text { and } \operatorname{st}_{K * L}(\sigma \cup \tau)=\operatorname{st}_{K}(\sigma) * \operatorname{st}_{L}(\tau)
$$

as subcomplexes of $K * L$, for every face $\sigma \in K$ and $\tau \in L$.

The vertices masquerade as algebraically independent variables of degree 2, and generate a graded polynomial algebra $S_{R}(V)$. For any subset $\omega \subseteq V$, we abbreviate the square-free monomial $\prod_{\omega} v_{i}$ to $v_{\omega}$, which has degree $2|\omega|$. We then define the Stanley-Reisner algebra $R[K]$ as $S_{R}(V) /\left(v_{\omega}: \omega \notin K\right)$; so the inclusions of the full subcomplexes $K, L \subset K * L$ induce an isomorphism

$$
R[K] \otimes R[L] \stackrel{\cong}{\longrightarrow} R[K * L] .
$$

Since $R[\Delta(\sigma)]=S_{R}(\sigma)$ for every face $\sigma$, an example is provided by

$$
S_{R}(\sigma) \otimes R\left[\ell_{K}(\sigma)\right] \stackrel{\cong}{\longrightarrow} R\left[\operatorname{st}_{K}(\sigma)\right]
$$

Any subcomplex $J \subseteq K$ induces a surjection $R[K] \rightarrow R[J]$, by annihilating the faces in $K \backslash J$; in particular, $K \subseteq \Delta(V)$ induces the canonical projection $S_{R}(V) \rightarrow R[K]$.

Every $K$ determines a finite category $\operatorname{CAT}(K)$, whose objects are the faces $\sigma$ and morphisms the inclusions $i_{\sigma, \tau}: \sigma \subseteq \tau$. The empty face is initial, so the classifying space $B \mathrm{CAT}(K)$ is contractible, whereas $B \mathrm{CAT}\left(K^{\times}\right)$is homeomorphic to the geometric realisation $|K|$. The maximal faces $\mu$ are characterised by the fact that they admit only identity morphisms. By construction, there is an isomorphism $\operatorname{CAT}(K) \times \operatorname{CAT}(L) \cong$ $\mathrm{CAT}(K * L)$ for any $K$ and $L$, which we use to identify $\operatorname{CAT}(\Delta(\sigma)) \times \operatorname{CAT}\left(\ell_{K}(\sigma)\right)$

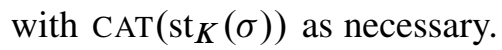

An A-diagram in an arbitrary category $\mathrm{R}$ consists of a covariant functor $D: \mathrm{A} \rightarrow \mathrm{R}$ for some small category A. 
Definitions 2.1 For any based CW-complex $(X, *)$, the CAT $(K)$-diagram

$$
X^{K}: \mathrm{CAT}(K) \longrightarrow \text { TOP }
$$

assigns the cartesian product $X^{\sigma}$ to each face $\sigma$, where $X^{K}(\varnothing)=*$; its value on $i_{\sigma, \tau}$ is the cofibration $X^{\sigma} \rightarrow X^{\tau}$, where the extra coordinates are set to $*$. The constant functor $\operatorname{cst}_{X^{V}}$ assigns $X^{V} \cong X^{m}$ to each face, and the identity map $\operatorname{id}_{X^{V}}$ to each inclusion. The natural transformation $i_{K}: X^{K} \rightarrow \operatorname{cst}_{X^{V}}$ is induced by the inclusions $i_{\sigma}: X^{\sigma} \rightarrow X^{V}$, for every face $\sigma$.

We are interested in two particular values of $X$, which stem from the inclusion of the cyclic subgroup $C$ in the circle $T$. To any subset $\sigma \subseteq V$, there corresponds the inclusion of the elementary abelian $p$-subgroup $C^{\sigma}$ in the torus $T^{\sigma}$, whose classifying map represents the projection of a product of infinite dimensional lens spaces onto the corresponding product of complex projective spaces. We shall denote this map by $t_{\sigma}: B C^{\sigma} \rightarrow B T^{\sigma}$, and assume that it is a homomorphism of abelian topological groups, written multiplicatively. It may be interpreted as a natural transformation

$$
t: B C^{K} \longrightarrow B T^{K} \text {. }
$$

The colimit $c(K):=\operatorname{colim}_{\mathrm{CAT}(K)} B T^{K}$ lies in the category $\mathrm{TOP}^{+}$of pointed topological spaces, and is a subcomplex of $B T^{V}$ via $i_{K}$.

Following Buchstaber and Panov [4], we view $c(K)$ as a distinguished representative for $\mathrm{DJ}(K)$. In [11, Section 3], this property is expressed in terms of isomorphisms

$$
H^{*}(c(K) ; R) \stackrel{\cong}{\longrightarrow} \lim S_{R}(K) \stackrel{\cong}{\longrightarrow} R[K],
$$

where $S_{R}(K)$ is the $\mathrm{CAT}^{\mathrm{op}}(K)$-diagram of graded commutative $R$-algebras whose value on $\sigma$ is $S_{R}(\sigma)$, and on $\tau \supseteq \sigma$ is the canonical projection $p_{\tau, \sigma}$.

In order to investigate the homotopy theoretical properties of $c(K)$, it is natural to consider the homotopy colimit hc $(K):=\operatorname{hocolim}_{\mathrm{CAT}(K)} B T^{K}$. Following Hollender and Vogt's elaboration [8] of the original definition of Bousfield and Kan [2], we describe $\mathrm{hc}(K)$ as the bar construction $B\left(*, \mathrm{CAT}(K), B T^{K}\right)$. As such, it lies in $\mathrm{TOP}^{+}$. The fact that $B T^{K}$ is cofibrant in the category of CAT $(K)$-diagrams [11, Lemma 2.7] ensures that the natural projection $\mathrm{hc}(K) \rightarrow c(K)$ is a homotopy equivalence, and that either space may be used as a model for $\mathrm{DJ}(K)$.

A deeper analysis of hc $(K)$ involves certain secondary structures associated to $\operatorname{CAT}(K)$, such as the undercategory $\sigma \downarrow \operatorname{CAT}(K)$. For any face $\sigma$ of $K$, this is obtained by restricting attention to those objects $\tau$ for which $\sigma \subseteq \tau$. The overcategory $\operatorname{CAT}(K) \downarrow \sigma$ 
is defined by analogy, and $(\sigma \downarrow \operatorname{CAT}(K))^{\mathrm{op}}$ is isomorphic to $\mathrm{CAT}^{\mathrm{op}}(K) \downarrow \sigma$. Setting $P(\rho):=\rho \backslash \sigma$ defines a functor

$$
P: \sigma \downarrow \operatorname{CAT}(K) \longrightarrow \operatorname{CAT}\left(\ell_{K}(\sigma)\right),
$$

which is also an isomorphism. Writing the restriction of $X^{K}$ to $\sigma \downarrow \operatorname{CAT}(K)$ as $X^{\sigma \downarrow K}$ and taking appropriate colimits then yields homeomorphisms

$$
\operatorname{colim} X^{\sigma \downarrow K} \equiv X^{\sigma} \times \operatorname{colim} X^{\ell_{K}(\sigma)} \equiv \operatorname{colim} X^{\mathrm{st}_{K}(\sigma)},
$$

where the former is induced by $P$. In case $X=B T$, we apply $H^{*}(-; R)$ and appeal to (4) to obtain isomorphisms

$$
\lim S_{R}(\sigma \downarrow \operatorname{CAT}(K)) \cong S_{R}(\sigma) \otimes R\left[\ell_{K}(\sigma)\right] \cong R\left[\operatorname{st}_{K}(\sigma)\right]
$$

of graded commutative algebras.

To organise our proofs, it is convenient to formalise the following, in which $X=X_{K}$ denotes a space that realises $\mathbb{Z}[K]$ for any choice of $K$.

Definitions 2.2 If an isomorphism $\theta_{X}: H^{*}(X ; \mathbb{Z}) \rightarrow \mathbb{Z}[K]$ is given, then the space $X$ reflects $K$, and $\theta_{X}$ is the reflector. If $\theta_{X}$ is given with coefficients $R$, then $X$ reflects $K$ over $R$.

For any subcomplex $J \subseteq K$, a map $e: W_{J} \rightarrow X_{K}$ reflects the pair $(K, J)$ whenever the square

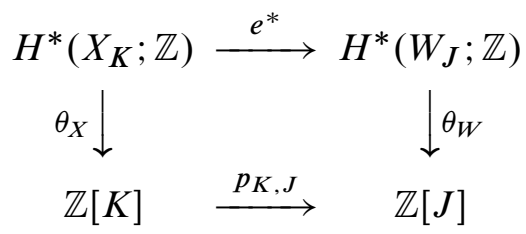

commutes, where $p_{K, J}$ denotes the canonical surjection.

For example, the classifying space $B T^{V}$ reflects $\Delta(V)$ by means of the standard isomorphism $H^{*}\left(B T^{V} ; \mathbb{Z}\right) \rightarrow S_{\mathbb{Z}}(V)$. More generally, the colimit $c(K)$ reflects $K$ for any complex $K$, and the inclusion $c(K) \subset B T^{V}$ reflects the pair $(\Delta(V) ; K)$. This may be extended to any other $X_{K}$ by interpreting $B T^{V}$ as an Eilenberg-Mac Lane space $H\left(\mathbb{Z}^{V}, 2\right)$. Then $\theta_{X}$ determines a unique homotopy class of maps $q_{X}: X_{K} \rightarrow B T^{V}$, which represents the $m$-tuple of generators in $H^{2}\left(X_{K}, \mathbb{Z}\right)$ and reflects $(\Delta(V), K)$ as before. Composing $q_{X}$ with projection onto $B T^{\sigma}$ creates a map $q_{\sigma}: X_{K} \rightarrow B T^{\sigma}$ for every face $\sigma$; its image in integral cohomology realises the polynomial subalgebra $\mathbb{Z}[\sigma]<\mathbb{Z}[K]$, via $\theta_{X}$. 
Canonical reflectors $\theta_{\mathrm{hc}(K)}: H^{*}(\mathrm{hc}(K)) \rightarrow \mathbb{Z}[K]$ arise by taking limits over $\mathrm{CAT}^{\mathrm{op}}(K)$, and have good functorial properties. So we may often interchange $H^{*}(c(K))$ and $\mathbb{Z}[K]$ without further comment.

Given any $e$ reflecting $(K, J)$, there is a homotopy commutative square

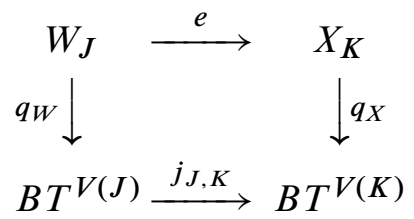

where $j_{J, K}$ denotes coordinatewise inclusion. This reflects the subpair

$$
(K, J) \subseteq(\Delta(V(K)), \Delta(V(J))),
$$

in the sense that it induces a commutative cube in cohomology when combined with the four reflectors.

For any noetherian local graded commutative algebra $A$ over $\mathbb{Q}$, a choice of homogeneous generators establishes a presentation

$$
0 \longrightarrow I_{A} \longrightarrow S \longrightarrow A \longrightarrow 0,
$$

where $S$ is a finitely generated graded polynomial algebra and $I_{A} \subset S$ is a graded ideal. Then $A$ is a complete intersection whenever $I_{A}$ is generated by a regular sequence of homogeneous elements; this definition is independent of the choice of generators by [3, Theorem 2.3.3]. We note in [11, Section 5] that the Stanley-Reisner algebra $\mathbb{Q}[K]$ is a complete intersection precisely when $K$ is an iterated join of the form $\Delta\left(U_{0}\right) * \partial\left(U_{1}\right) * \cdots * \partial\left(U_{t}\right)$, for any partition $\left\{U_{0}, U_{1}, \ldots, U_{t}\right\}$ of $V$. In this case, an isomorphism

$$
S_{\mathbb{Q}}\left(U_{0}\right) \otimes \mathbb{Q}\left[\partial\left(U_{1}\right)\right] \otimes \cdots \otimes \mathbb{Q}\left[\partial\left(U_{t}\right)\right] \cong \mathbb{Q}[K]
$$

is given by (1) above.

Theorem 9.4 Let $X$ be a nilpotent $C W$-complex, and suppose that $\mathbb{Q}[K]$ is a complete intersection; then there is an isomorphism $\theta: H^{*}(X ; \mathbb{Z}) \rightarrow \mathbb{Z}[K]$ if and only if there is a homotopy equivalence $f: \operatorname{hc}(K) \rightarrow X$ such that $f^{*}=\theta$.

We prove Theorem 9.4 by using the arithmetic square to combine the rational results of [11] with the following $p$-adic statement, which refers to a larger class of complexes.

Theorem 8.6 Let $X$ be a $p$-complete $C W$-complex, and suppose that $K$ is an iterated join $\Delta^{\left(r_{1}\right)}\left(U_{1}\right) * \cdots * \Delta^{\left(r_{t}\right)}\left(U_{t}\right)$ of skeleta of simplices; then there is an isomorphism $\theta: H^{*}\left(X ; \mathbb{Z}_{p}^{\wedge}\right) \rightarrow \mathbb{Z}_{p}^{\wedge}[K]$ if and only if there is a homotopy equivalence $f: \operatorname{hc}(K)_{p}^{\wedge} \rightarrow X$ such that $f^{*}=\theta$. 
After suspension, however, the situation becomes much simpler.

Theorem 9.5 Let $X$ be a $C W$-complex that realises $\mathbb{Z}[K]$ for any $K$; then there is a homotopy equivalence $e: \Sigma X \rightarrow \Sigma$ hc $(K)$.

Our final result on completions is a homotopy classification of self-equivalences of hc $(K)$. We do not expect a global version to hold, even under the assumptions of Theorem 9.4.

Theorem 8.7 For complexes $K$ as in Theorem 8.6, any pair of self-equivalences $f, g: \operatorname{hc}(K)_{p}^{\wedge} \rightarrow \operatorname{hc}(K)_{p}^{\wedge}$ are homotopic if and only if $H^{*}\left(f ; \mathbb{Z}_{p}^{\wedge}\right)=H^{*}\left(g ; \mathbb{Z}_{p}^{\wedge}\right)$.

For these $K$, it is also of interest to apply [7, Theorem 10.2(3)], and deduce that the $t$-fold suspension $\Sigma^{t} \mathcal{Z}_{K}$ of the moment-angle complex [5; 4] is homotopy equivalent to a wedge of spheres.

\section{Stanley-Reisner algebras and the $T$-functor}

We begin by focusing our attention on the $\bmod p$ Stanley-Reisner algebra $\mathbb{F}_{p}[K]$, where $K$ is a specific simplicial complex with generic face $\sigma$. It is convenient to denote $H^{*}\left(-; \mathbb{F}_{p}\right)$ by $H^{*}(-)$ throughout this section, and to abbreviate $H^{*}(B E)$ to $H E$ for an arbitrary elementary abelian $p$-group $E$. Often, $E$ will be of the form $C^{\sigma}$ for some face $\sigma$ of $K$.

For any space $X$ reflecting $K$ over $\mathbb{F}_{p}$, our aim is to construct maps $B E \rightarrow X$ with prescribed cohomological properties. We proceed by studying the mapping space $\operatorname{map}(B E, X)$, and take advantage of the fact that its mod $p$ cohomology ring may be computed by Lannes' functor $T_{E}$. We refer to Lannes [10] and Schwartz [13] for the basic properties of $T_{E}$, which we summarise below. We follow their notation by writing $\mathcal{U}$ for the category of unstable modules over the mod $p$ Steenrod algebra $\mathcal{A}_{p}$, and $\mathcal{K}$ for the subcategory of unstable algebras over $\mathcal{A}_{p}$. In order to avoid technical difficulties, we shall only consider algebras that are finitely generated; thus $\mathcal{K}\left(A, A^{\prime}\right)$ is a finite set for any algebras $A$ and $A^{\prime}$.

By definition, $T_{E}$ is left adjoint to the functor $H E \otimes-: \mathcal{U} \rightarrow \mathcal{U}$. The latter is compatible with the forgetful functor $\mathcal{K} \rightarrow \mathcal{U}$, and $T_{E}: \mathcal{K} \rightarrow \mathcal{K}$ is left adjoint to its restriction. By construction, $T_{C}$ coincides with the $n$-fold iterate $T_{C} \cdots T_{C}$ for any $n$, and $T_{E}$ preserves tensor products in $\mathcal{U}$ and $\mathcal{K}$. 
For any space $Y$, evaluation defines a map $B E \times \operatorname{map}(B E, Y) \rightarrow Y$. Passing to cohomology and left adjointing yields a $\mathcal{K}$-morphism

$$
a_{Y}: T_{E} H^{*}(Y) \longrightarrow H^{*}(\operatorname{map}(B E, Y)),
$$

which is an isomorphism whenever $Y$ is $p$-complete and $T_{E} H^{*}(Y)$ has finite type and vanishes in degree 1 . So $T_{E}$ computes the $\bmod p$ cohomology of the mapping space under these conditions [10, Theorem 3.2.1].

The splitting of $\operatorname{map}(B E, Y)$ into the disjoint union $\bigsqcup \operatorname{map}(B E, Y)_{f}$ of its connected components is mirrored by the algebraic properties of $T_{E}$, as follows. For each unstable algebra $A$ and $\mathcal{K}$-morphism $\phi: A \rightarrow H E$, its adjoint restricts to a map $T_{E}^{0} A \rightarrow \mathbb{F}_{p}$ on the zero-dimensional part. We write $\mathbb{F}_{p}(\phi)$ when it is necessary to emphasise the induced $T_{E}^{0} A$-module structure on $\mathbb{F}_{p}$. The component of $T_{E} A$ associated to $\phi$ is defined by

$$
T_{E}(A, \phi):=T_{E} A \otimes_{T_{E}^{0} A} \mathbb{F}_{p}(\phi),
$$

and Lannes' linearisation principle [13, Theorem 3.8.6] leads to splittings

$$
T_{E} A \cong \bigoplus_{\phi \in \mathcal{K}(A, H E)} T_{E}(A, \phi) \quad \text { and } \quad T_{E}^{0} A \cong \bigoplus_{\phi \in \mathcal{K}(A, H E)} \mathbb{F}_{p}(\phi)
$$

In particular, we may identify $T_{E}^{0} A$ with the $p$-Boolean algebra $\mathbb{F}_{p}^{\mathcal{K}(A, H E)}$.

In the case that $A=H^{*}(Y)$ and $\phi=f^{*}$ for some $f: B E \rightarrow Y$, the morphism $a_{Y}$ of (11) restricts to a $\mathcal{K}$-morphism

$$
a_{f}: T_{E}\left(H^{*}(Y), f^{*}\right) \longrightarrow H^{*}\left(\operatorname{map}(B E, Y)_{f}\right) .
$$

Since the algebraic components of $T_{E} H^{*}(Y)$ correspond bijectively to the connected components of map $(B E, Y))$, it follows that $a_{Y}$ splits accordingly.

The following key example is based on the proof of [13, Proposition 9.8], in which $\operatorname{map}\left(B E, B T^{\omega}\right)$ is identified with $E(\omega) \times B T^{\omega}$, where $E(\omega)$ denotes the discrete group of homomorphisms $E \rightarrow E^{\omega}$ for any subset $\omega \subseteq V$.

Example 3.1 As $\mathcal{A}_{p}$-algebras, $H T^{\omega}:=H^{*}\left(B T^{\omega}\right)$ is canonically isomorphic to $S_{\mathbb{F}_{p}}(\omega)$, and multiplication on $B T^{\omega}$ induces a coproduct $d$. The $\mathcal{K}$-morphisms $\phi: H T^{\omega} \rightarrow H E$ correspond bijectively to group homomorphisms $E \rightarrow T^{\omega}$, and hence to $E(\omega)$. The coaugmentation $c_{\phi}: H T^{\omega} \rightarrow T_{E}\left(H T^{\omega}, \phi\right)$ is an isomorphism in $\mathcal{K}$, whose inverse $r_{\phi}$ is the adjoint of $(\phi \otimes 1) \circ d$.

For each $f: B E \rightarrow B T^{\omega}$, the composition

$$
H T^{\omega} \stackrel{c_{f}}{\longrightarrow} T_{E}\left(H T^{\omega}, f^{*}\right) \stackrel{a_{f}}{\longrightarrow} H^{*}\left(\operatorname{map}\left(B E, B T^{\omega}\right)_{f}\right)
$$


computes the mod $p$ cohomology of the mapping space, and is induced by evaluation $\operatorname{map}\left(B E, B T^{\omega}\right)_{f} \rightarrow B T^{\omega}$ at the base point. Its inverse is induced by the adjoint of the action $s_{f}: B E \times B T^{\omega} \rightarrow B T^{\omega}$, defined by $(b, u) \mapsto u f(b)$.

For any $\mathcal{K}$-morphism $\pi: A_{1} \rightarrow A_{2}$, the induced homomorphism

$$
T_{E}^{0}(\pi): \bigoplus_{\phi \in \mathcal{K}\left(A_{1}, H E\right)} \mathbb{F}_{p}(\phi) \cong T_{E}^{0} A_{1} \longrightarrow T_{E}^{0} A_{2} \cong \bigoplus_{\psi \in \mathcal{K}\left(A_{2}, H E\right)} \mathbb{F}_{p}(\psi)
$$

is determined by composition $\circ \pi: \mathcal{K}\left(A_{2}, H E\right) \rightarrow \mathcal{K}\left(A_{1}, H E\right)$ with $\pi$. For any $\phi \in \mathcal{K}\left(A_{1}, H E\right)$, we denote the subset $(\circ \pi)^{-1}(\phi) \subset \mathcal{K}\left(A_{2}, H E\right)$ by $\operatorname{ext}(\phi, \pi)$; its elements $\psi$ satisfy $\psi \circ \pi=\phi$. We may then identify $T_{E}^{0}(\pi)$ with the direct sum of diagonal maps of the form $\mathbb{F}_{p}(\phi) \rightarrow \bigoplus_{\psi \in \operatorname{ext}(\phi, \pi)} \mathbb{F}_{p}(\psi)$, and obtain an isomorphism

$$
T_{E}^{0} A_{2} \otimes_{T_{E}^{0} A_{1}} \mathbb{F}_{p}(\phi) \cong \bigoplus_{\psi \in \operatorname{ext}(\phi, \pi)} \mathbb{F}_{p}(\psi)
$$

For any unstable algebra $A$ in $\mathcal{K}$ we write $A-\mathcal{U}$ for the category whose objects $M$ are simultaneously $A$-modules and unstable $\mathcal{A}_{p}$-modules, and whose structure maps $A \otimes M \rightarrow M$ are $\mathcal{A}_{p}$-linear; here $\mathcal{A}_{p}$ acts on $A \otimes M$ via the Cartan formula. The morphisms in $A-\mathcal{U}$ are both $A$ - and $\mathcal{A}_{p}$-linear. Since the $T$-functor commutes with tensor products, $T_{E}$ extends to a functor $A-\mathcal{U} \rightarrow\left(T_{E} A\right)-\mathcal{U}$, which we also label $T_{E}$. For every morphism $\phi: A \rightarrow H E$, we may then define the relative component

$$
T_{E}(M, \phi):=T_{E} M \otimes_{T_{E}^{0} A} \mathbb{F}_{p}(\phi)
$$

by analogy with (12), and obtain a splitting $T_{E} M \cong \bigoplus_{\phi \in \mathcal{K}(A, H E)} T_{E}(M, \phi)$. Furthermore, the coaugmentation $A \rightarrow T_{E}(A, \phi)$ makes $T_{E}(M, \phi)$ into an $A$-module, and $T_{E}(-, \phi)$ becomes an endofunctor on $A-\mathcal{U}$.

In particular, the morphism $\pi$ of (15) turns $A_{2}$ into an object of $A_{1}-\mathcal{U}$, where there are isomorphisms

$$
\begin{aligned}
T_{E}\left(A_{2}, \phi\right) & \cong T_{E} A_{2} \otimes_{T_{E}^{0} A_{1}} \mathbb{F}_{p}(\phi) \\
& \cong T_{E} A_{2} \otimes_{T_{E}^{0} A_{2}} T_{E}^{0} A_{2} \otimes_{T_{E}^{0} A_{1}} \mathbb{F}_{p}(\phi) \\
& \cong T_{E} A_{2} \otimes_{T_{E}^{0} A_{2}}\left(\bigoplus_{\psi \in \operatorname{ext}(\phi, \pi)} \mathbb{F}_{p}(\psi)\right) \\
& \cong \bigoplus_{\psi \in \operatorname{ext}(\phi, \pi)} T_{E}\left(A_{2}, \psi\right) .
\end{aligned}
$$

One additional property of the $T$-functor is that $T_{E}$ commutes with finite limits; in other words, for any finite category $\mathrm{C}$ and diagram $F: \mathrm{C} \rightarrow \mathcal{K}$, there exists a $\mathcal{K}-$ isomorphism $T_{E}(\lim F) \cong \lim T_{E} F$. In this situation, we are interested in computing the components of $T_{E}(\lim F)$. To avoid technical difficulties we assume that $F(c)$ is finitely generated as an algebra for every object $c$ of $\mathrm{C}$, and similarly for $A:=\lim F$. 
The limit is defined by an exact sequence of the form

$$
0 \longrightarrow A \longrightarrow \prod_{c} F(c) \stackrel{\delta}{\longrightarrow} \prod_{c_{0} \rightarrow c_{1}} F\left(c_{1}\right),
$$

and the natural projections $\pi_{c}: A \rightarrow F(c)$ turn $\delta$ into an $A-\mathcal{U}$-morphism. For every $\phi: A \rightarrow H E$ the functor $T_{E}(-, \phi)$ is exact on $A-\mathcal{U}$, because it is a retract of $T_{E}$. We therefore obtain an exact sequence

$$
0 \longrightarrow T_{E}(A, \phi) \longrightarrow \prod_{c} T_{E}(F(c) ; \phi) \longrightarrow \prod_{c_{0} \rightarrow c_{1}} T_{E}\left(F\left(c_{1}\right), \phi\right),
$$

which identifies $T_{E}\left(\lim _{\mathrm{C}} F, \phi\right)$ with $\lim _{\mathrm{C}} T_{E}(F, \phi)$.

If $\operatorname{ext}\left(\phi, \pi_{c}\right)$ is empty, then $T_{E}(F(c), \phi)=0$. So we consider the full subcategory $\mathrm{C}\langle F, \phi\rangle$ of $\mathrm{C}$, whose objects are defined by insisting that $\operatorname{ext}\left(\phi, \pi_{c}\right)$ be nonempty. For any morphism $c^{\prime} \rightarrow c$ with $c$ in $\mathrm{C}\langle F, \phi\rangle$, it follows that $c^{\prime}$ also lies in $\mathrm{C}\langle F, \phi\rangle$; so there exists an isomorphism

$$
T_{E}\left(\lim _{\mathrm{C}} F, \phi\right) \cong \lim _{\mathrm{C}\langle F, \phi\rangle} T_{E}(F, \phi) .
$$

We apply these considerations to the diagram $S_{\mathbb{F}_{p}}(K): \mathrm{CAT}^{\mathrm{op}}(K) \rightarrow \mathcal{K}$ of $(4)$, whose ob-

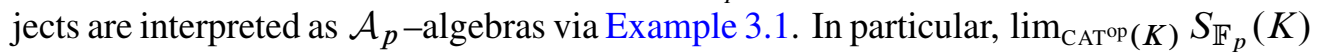
is isomorphic to $\mathbb{F}_{p}[K]$, and the $\mathcal{K}$-morphism $\pi_{\sigma}: \mathbb{F}_{p}[K] \rightarrow S_{\mathbb{F}_{p}}(\sigma)$ is the standard projection, for every $\sigma \in K$. We focus on the $\mathcal{K}$-morphisms $\phi_{\sigma}: \mathbb{F}_{p}[K] \rightarrow H C^{\sigma}$, defined in terms of (3) by $t_{\sigma}^{*} \circ \pi_{\sigma}$.

Proposition 3.1 For any face $\sigma$ of $K$, there is an isomorphism

$$
r_{\sigma}: T_{C^{\sigma}}\left(\mathbb{F}_{p}[K], \phi_{\sigma}\right) \longrightarrow \mathbb{F}_{p}\left[\operatorname{st}_{K}(\sigma)\right]
$$

as objects of $\mathcal{K}$.

Proof The set $\operatorname{ext}\left(\phi_{\sigma}, \pi_{\tau}\right)$ contains the single element $\phi_{\tau, \sigma}: S_{\mathbb{F}_{p}}(\tau) \rightarrow H C^{\sigma}$, defined by $t_{\sigma}^{*} \circ p_{\tau, \sigma}$, if and only if $\sigma \subseteq \tau$. Otherwise, $\operatorname{ext}\left(\phi_{\sigma}, \pi_{\tau}\right)$ is empty; so for any particular $\sigma$, we may identify $\operatorname{CAT}(K)\left\langle S_{\mathbb{F}_{p}}(K), \phi_{\sigma}\right\rangle$ with $\sigma \downarrow \operatorname{CAT}(K)$.

For $\sigma \subseteq \tau$, Example 3.1 provides isomorphisms

$$
r_{\sigma}(\tau): T_{C^{\sigma}}\left(S_{\mathbb{F}_{p}}(\tau), \phi_{\tau, \sigma}\right) \longrightarrow S_{\mathbb{F}_{p}}(\tau)
$$

that are functorial in $\tau$, and so define an isomorphism

$$
r_{\sigma}(K): T_{C^{\sigma}}\left(S_{\mathbb{F}_{p}}(K), \phi_{\sigma}\right) \longrightarrow S_{\mathbb{F}_{p}}(\sigma \downarrow \operatorname{CAT}(K))
$$

of $\operatorname{CAT}^{\mathrm{op}}(K) \downarrow \sigma$-diagrams in $\mathcal{K}$. Then take limits, using (7) and (16). 
Corollary 3.2 For every maximal face $\mu$ of $K$, there exists a $\mathcal{K}$-isomorphism $r_{\mu}: T_{C^{\mu}}\left(\mathbb{F}_{p}[K], \phi_{\mu}\right) \rightarrow S_{\mathbb{F}_{p}}(\mu)$.

Proof Since $\mu$ is maximal, its star is the simplex $\Delta(\mu)$.

We now introduce the crucial map $f_{\sigma}: B C^{\sigma} \rightarrow X$, and prove the main results of this section. It is convenient to write the space $\operatorname{map}\left(B C^{\sigma}, X\right)_{f_{\sigma}}$ as $m^{\sigma}(X)$, and evaluation at the identity of $B C^{\sigma}$ as ev: $m^{\sigma}(X) \rightarrow X$.

Theorem 3.3 If $X$ is $p$-complete and reflects $K$ by means of an isomorphism $\theta: H^{*}(X) \rightarrow \mathbb{F}_{p}[K]$, then

(1) there exists a map $f_{\sigma}: B C^{\sigma} \rightarrow X$ such that $f_{\sigma}^{*}=\phi_{\sigma} \circ \theta$, and it is uniquely determined up to homotopy;

(2) the space $m^{\sigma}(X)$ is $p$-complete, and reflects st $K(\sigma)$ over $\mathbb{F}_{p}$;

(3) the map ev: $m^{\sigma}(X) \rightarrow X$ reflects $\left(K\right.$, st $\left._{K}(\sigma)\right)$ over $\mathbb{F}_{p}$;

(4) for maximal $\mu$, there is a homotopy equivalence $m^{\mu}(X) \simeq\left(B T^{\mu}\right)_{p}^{\wedge}$.

Proof (1) Every $\mathcal{K}$-morphism $H^{*}(X) \rightarrow H C^{\sigma}$ may be realised uniquely up to homotopy by [10, Théorème 3.1.1], because $X$ is $p$-complete and $H^{*}(X)$ has finite type.

(2) By [10, Proposition 3.4.4], $m^{\sigma}(X)$ inherits the property of completeness from $X$. Also, $T_{C^{\sigma}}\left(H^{*}(X), \phi_{\sigma}\right)$ is of finite type and vanishes in degree 1, by Proposition 3.1. So $a_{\sigma}:=a_{f_{\sigma}}: T_{C^{\sigma}}\left(H^{*}(X), \phi_{\sigma}\right) \rightarrow H^{*}\left(m^{\sigma}(X)\right)$ of (13) is an isomorphism, and the required reflector is $\theta_{m^{\sigma}(X)}:=r_{\sigma} \circ T_{C^{\sigma}}(\theta) \circ a_{\sigma}^{-1}$.

(3) By construction, ev*: $H^{*}(X) \rightarrow H^{*}\left(m^{\sigma}(X)\right)$ is the composition of $f_{\sigma}^{*}$ with the coaugmentation $c_{\sigma}$ for $T_{C^{\sigma}}$. So we may combine (2) with the functorial properties of $T$ to obtain a commutative ladder

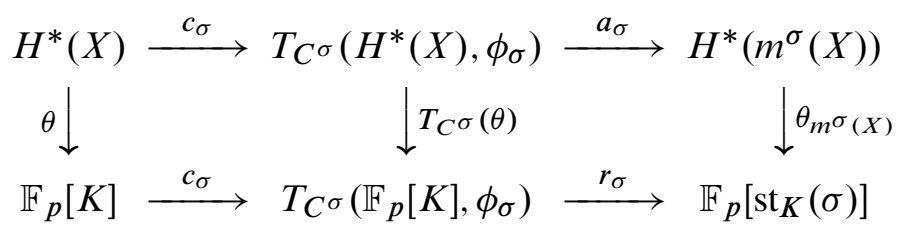

whose outer rectangle is a commutative square of the form (8); hence ev is the required reflector.

(4) It suffices to combine (2), Corollary 3.2, and the fact that the homotopy type of $\left(B T^{\mu}\right)_{p}^{\wedge}$ is determined by its mod $p$ cohomology. 
Remarks 3.2 (1) The mod $p$ cohomology of $X$ of Theorem 3.3 is concentrated in even dimensions, so $H^{\mathrm{ev}}\left(X ; \mathbb{Z}_{p}^{\wedge}\right)$ is torsion free and $H^{\text {od }}\left(X ; \mathbb{Z}_{p}^{\wedge}\right)$ is zero. Since Theorem 3.3(3) implies that evaluation induces an epimorphism in mod $p$ cohomology, the same must hold over $\mathbb{Z}_{p}^{\wedge}$.

(2) We may replace $\phi_{\sigma}$ in Proposition 3.1 with the homomorphism induced by the constant map cst: $B C^{\sigma} \rightarrow X$, which we write as $\kappa_{\sigma}: \mathbb{F}_{p}[K] \rightarrow H C^{\sigma}$. Then $\mathrm{C}\left\langle F, \kappa_{\sigma}\right\rangle=\mathrm{C}$, and we obtain an isomorphism $T_{C^{\sigma}}\left(\mathbb{F}_{p}[K], \kappa_{\sigma}\right) \rightarrow \mathbb{F}_{p}[K]$. It follows that ev: $\operatorname{map}\left(B C^{\sigma}, X\right)_{\mathrm{cst}} \rightarrow X$ induces an isomorphism in mod $p$ cohomology, by adapting the proofs of Theorem 3.3(2) and (3); it is therefore an equivalence, because the source and target are $p$-complete.

\section{Mapping spaces and p-adic cohomology: I}

In this section we extend our calculations to $p$-adic cohomology for the mapping space $m^{\sigma}(X)$ of Theorem 3.3(2). We insist that $X$ is $p$-complete and 1-connected, and reflects $K$ over $\mathbb{Z}_{p}^{\wedge}$ by means of an isomorphism $\theta: H^{*}\left(X ; \mathbb{Z}_{p}^{\wedge}\right) \rightarrow \mathbb{Z}_{p}^{\wedge}[K]$. The reduction of $\theta \bmod p$ is therefore a reflector over $\mathbb{F}_{p}$, and Theorem 3.3 applies.

For our first result, we consider the evaluation map of Theorem 3.3(3), and abbreviate $V\left(\operatorname{st}_{K}(\sigma)\right)$ to $V_{K}(\sigma)$ throughout.

Lemma 4.1 For any face $\sigma \in K$, the map $q_{m^{\sigma}(X)}$ may be chosen so as to make the diagram

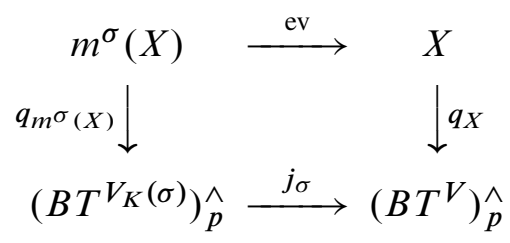

homotopy commutative, where $j_{\sigma}$ denotes coordinatewise inclusion.

Proof Define $q_{m^{\sigma}(X)}$ by composing $q_{X} \circ \mathrm{ev}$ with projection onto $\left(B T^{V_{K}(\sigma)}\right)_{p}^{\wedge}$. The diagram induced by (18) in mod $p$ cohomology is then commutative, by Theorem 3.3(3), and it remains to prove that $\operatorname{ev}^{*} q_{X}^{*}\left(v_{i}\right)$ is also zero in $H^{2}\left(m^{\sigma}(X) ; \mathbb{Z}_{p}^{\wedge}\right)$ for any vertex $v_{i} \notin V_{K}(\sigma)$.

Let $q_{i}: X \rightarrow B T_{p}^{\wedge}$ represent $v_{i}$. The Serre spectral sequence of the induced spherical fibration

$$
T_{p}^{\wedge} \longrightarrow F_{i} \longrightarrow X
$$


shows that $H^{*}\left(F_{i} ; \mathbb{F}_{p}\right)$ has finite type, and $F_{i}$ is $p$-complete because $q_{i}$ is a map of $1-$ connected $p$-complete spaces. Also, $q_{i} \circ f_{\sigma}: B C^{\sigma} \rightarrow B T_{p}^{\wedge}$ is null-homotopic for any $v_{i} \notin V_{K}(\sigma)$, so $f_{\sigma}$ lifts to a map $f_{i}: B C^{\sigma} \rightarrow F_{i}$. By [15], the functor map $\left(B C^{\sigma},-\right)$ converts $F_{i} \rightarrow X \rightarrow B T_{p}^{\wedge}$ into a fibration

$$
\operatorname{map}\left(B C^{\sigma}, F_{i}\right)_{f_{i}} \stackrel{g_{i}}{\longrightarrow} m^{\sigma}(X) \stackrel{k_{i}}{\longrightarrow} \operatorname{map}\left(B C^{\sigma}, B T_{p}^{\wedge}\right)_{\mathrm{cst}},
$$

whose base is homotopy equivalent to $B T_{p}^{\wedge}$ by Example 3.1. We abbreviate the fibre to $m^{\sigma}\left(F_{i}\right)$, and note that it is path connected and $p$-complete, because $k_{i}$ is a map of 1-connected $p$-complete spaces; and $H^{*}\left(m^{\sigma}\left(F_{i}\right) ; \mathbb{F}_{p}\right)$ is of finite type, because the same holds for $H^{*}\left(m^{\sigma}(X) ; \mathbb{F}_{p}\right)$. We may then deduce from [10, Proposition 3.4.4] that $T_{C^{\sigma}}$ computes $H^{*}\left(m^{\sigma}\left(F_{i}\right) ; \mathbb{F}_{p}\right)$. But the Gysin sequence of (19) confirms that $H^{*}\left(F_{i} ; \mathbb{Z}_{p}^{\wedge}\right)$ is torsion-free, so the same is also true for $H^{*}\left(m^{\sigma}\left(F_{i}\right) ; \mathbb{Z}_{p}^{\wedge}\right)$ by $[9$, Theorem A].

Now $k_{i}^{*} v_{i} \in H^{2}\left(m^{\sigma}(X) ; R\right)$ is the Euler class $e\left(k_{i}\right)$ of the principal $T_{p}^{\wedge}$-fibration $k_{i}$ for any coefficients $R$, and equals $\mathrm{ev}^{*} q_{X}^{*}\left(v_{i}\right)$ by construction. Thus $e\left(k_{i}\right)$ vanishes mod $p$ for every $v_{i} \notin V\left(\mathrm{st}_{K}(\sigma)\right)$, and we need only prove that the same is true $p$-adically. The mod $p$ Gysin sequence shows that $g_{i}^{*}: H^{*}\left(m^{\sigma}(X) ; \mathbb{F}_{p}\right) \rightarrow$ $H^{*}\left(m^{\sigma}\left(F_{i}\right) ; \mathbb{F}_{p}\right)$ is a monomorphism; and there are isomorphisms $H^{*}\left(Y ; \mathbb{F}_{p}\right) \cong$ $H^{*}\left(Y ; \mathbb{Z}_{p}^{\wedge}\right) \otimes \mathbb{F}_{p}$ for $Y=m^{\sigma}(X)$ and $m^{\sigma}\left(F_{i}\right)$, because $H^{*}\left(Y ; \mathbb{Z}_{p}^{\wedge}\right)$ is torsion-free in both cases. Then Nakayama's lemma confirms that $g_{i}^{*}$ is also a $p$-adic monomorphism, and $e\left(k_{i}\right)=0$ as sought.

Corollary 4.2 For any maximal face $\mu \in K$, the map $q_{m^{\mu}(X)}$ is a homotopy equivalence and determines a homotopy commutative square

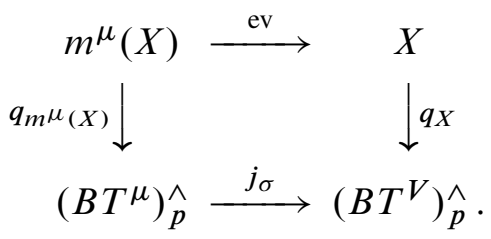

Proof The statement follows from Lemma 4.1 by setting $\sigma=\mu$, and identifying $q_{m^{\mu}(X)}$ as a homotopy equivalence by Theorem 3.3(4).

Definition 4.3 For any maximal face $\mu$, the map $h_{\mu}: B T^{\mu} \rightarrow X$ is the composition of ev $\circ q_{m^{\mu}(X)}^{-1}$ with completion. For any other face $\sigma$, the map $h_{\sigma}: B T^{\sigma} \rightarrow X$ is $h_{\mu} \circ i_{\sigma, \mu}$ for some choice of $\mu \supseteq \sigma$. 
Remarks 4.1 (1) In Section 5, we shall show that the homotopy class of $h_{\sigma}$ is independent of the choice of $\mu$, and therefore that the diagram

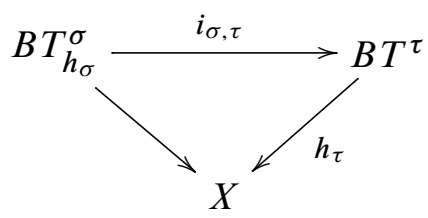

is homotopy commutative for every $\sigma \subset \tau$. In fact $h_{\mu}$ reflects the pair $(K, \Delta(\mu))$ over $\mathbb{Z}_{p}^{\wedge}$ by construction, so the diagram induced by (20) is certainly commutative in $p$-adic cohomology.

(2) In [6], Dehon and Lannes study maps $B T^{r} \rightarrow Y$ for spaces such that $H^{*}(Y ; \mathbb{Z})$ is torsion free and concentrated in even degrees. They deduce that $H^{*}(-; \mathbb{Z})$ classifies such maps up to homotopy. Their proof establishes a similar result for the $p$-adic cohomology of $p$-complete spaces, and may also be adapted to show that the homotopy class of $h_{\sigma}$ is independent of $\mu$.

(3) For any face $\sigma$, Theorem 3.3(1) provides a homotopy

$$
h_{\sigma} \circ t_{\sigma} \simeq f_{\sigma}: B C^{\sigma} \longrightarrow X .
$$

Theorem 4.4 If $X$ is $p$-complete and reflects $K$ over $\mathbb{Z}_{p}^{\wedge}$, then

(1) the space $m^{\sigma}(X)$ reflects st $_{K}(\sigma)$ over $\mathbb{Z}_{p}^{\wedge}$;

(2) the map ev: $m^{\sigma}(X) \rightarrow X$ reflects the pair $\left(K\right.$, st $\left._{K}(\sigma)\right)$ over $\mathbb{Z}_{p}^{\wedge}$.

Proof (1) For any pair of faces $\sigma \subseteq \tau$, write $i_{\sigma, \tau} \circ t_{\sigma}$ as $t_{\sigma, \tau}: B C^{\sigma} \rightarrow B T^{\tau}$. Then (21) confirms that $h_{\tau} \circ t_{\sigma, \tau}: B C^{\sigma} \rightarrow X$ is homotopic to $f_{\sigma}$. Passing to mapping spaces and employing Example 3.1 defines a composition

$$
l_{\sigma, \tau}: B T^{\tau} \stackrel{e_{\sigma, \tau}}{\longrightarrow} \operatorname{map}\left(B C^{\sigma}, B T^{\tau}\right)_{t_{\sigma, \tau}} \stackrel{h_{\tau} \circ}{\longrightarrow} m^{\sigma}(X),
$$

where $e_{\sigma, \tau}(u)$ acts by $b \mapsto u t_{\sigma, \tau}(b)$, and evol $l_{\sigma, \tau} \simeq h_{\tau}$. Since $H^{*}\left(\mathrm{ev} ; \mathbb{Z}_{p}^{\wedge}\right)$ is epic by Remarks 3.2(1), all choices of $h_{\tau}$ induce the same homomorphism $H^{*}\left(l_{\sigma, \tau} ; \mathbb{Z}_{p}^{\wedge}\right)$. So for any $\sigma \subseteq \tau \subseteq \rho$ we obtain a commutative triangle

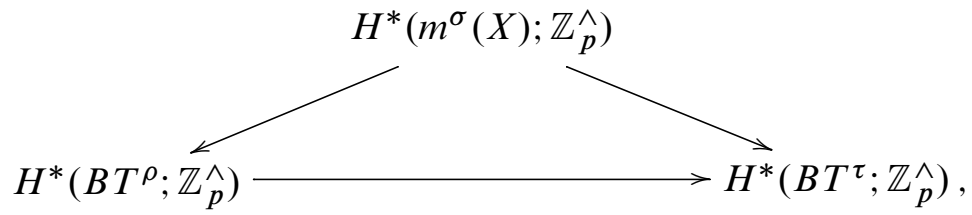


thus defining a $\left(\mathrm{CAT}^{\mathrm{op}}(K) \downarrow \sigma\right)$-diagram $H T^{\sigma \downarrow K}$ of graded commutative $\mathbb{Z}_{p}^{\wedge}$-algebras, and an induced homomorphism

$$
\theta^{\prime}: H^{*}\left(m^{\sigma}(X) ; \mathbb{Z}_{p}^{\wedge}\right) \longrightarrow \lim H T^{\sigma \downarrow K} .
$$

By (7), the target is isomorphic to $\mathbb{Z}_{p}^{\wedge}\left[\operatorname{st}_{K}(\sigma)\right]$.

Now consider the homomorphism $\theta^{\prime \prime}: H^{*}\left(m^{\sigma}(X) ; \mathbb{F}_{p}\right) \rightarrow \mathbb{F}_{p}\left[\mathrm{st}_{K}(\sigma)\right]$, given by reducing $\theta^{\prime} \bmod p$; it may be identified with the isomorphism $\theta_{m^{\sigma}(X)}$ of (17) by noting the commutativity of the square

$$
\begin{array}{ccc}
T_{C^{\sigma}}\left(H^{*}\left(X ; \mathbb{F}_{p}\right), \phi_{\sigma}\right) & \stackrel{a_{\sigma}}{\longrightarrow} & H^{*}\left(m^{\sigma}(X) ; \mathbb{F}_{p}\right) \\
T_{C} \sigma\left(\pi_{\tau} \circ \theta\right) \downarrow & & l_{\sigma, \tau}^{*} \\
T_{C^{\sigma}}\left(S_{\mathbb{F}_{p}}(\tau), \phi_{\tau, \sigma}\right) & \stackrel{r_{\sigma}(\tau)}{\longrightarrow} & S_{\mathbb{F}_{p}}(\tau)
\end{array}
$$

for any $\tau \supseteq \sigma$, and taking limits over $\mathrm{CAT}^{\mathrm{op}}(K) \downarrow \sigma$. Applying the exact sequence $\mathbb{Z}_{p}^{\wedge} \stackrel{p}{\rightarrow} \mathbb{Z}_{p}^{\wedge} \rightarrow \mathbb{F}_{p}$ yields a commutative ladder of short exact sequences

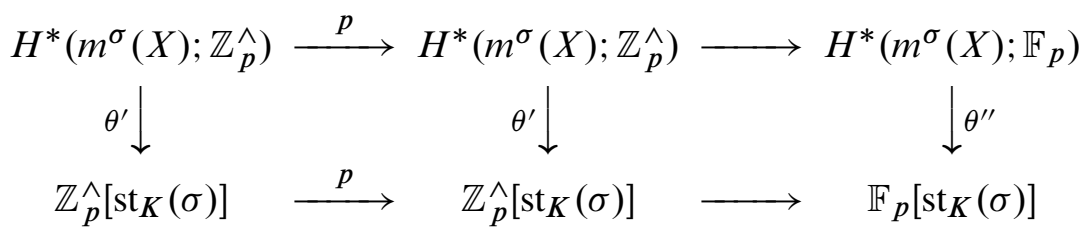

because $H^{*}\left(m^{\sigma}(X) ; \mathbb{Z}_{p}^{\wedge}\right)$ is free. But $\theta^{\prime \prime}$ is an isomorphism, so $\theta^{\prime}$ is also an isomorphism by the snake lemma, and is therefore the required reflector.

(2) The homomorphism ev*: $H^{*}\left(X ; \mathbb{Z}_{p}^{\wedge}\right) \rightarrow H^{*}\left(m^{\sigma}(X) ; \mathbb{Z}_{p}^{\wedge}\right)$ induces morphisms of triangles (22) over the inclusion functor $\sigma \downarrow \operatorname{CAT}(K) \rightarrow \operatorname{CAT}(K)$. The required square commutes, by Lemma 4.1 .

Corollary 4.5 If $\mathrm{st}_{K}(\sigma)$ coincides with $K$, then ev: $m^{\sigma}(X) \rightarrow X$ is a homotopy equivalence and the reflectors satisfy $\theta^{\prime} \circ \mathrm{ev}^{*}=\theta_{X}$ over $\mathbb{Z}_{p}^{\wedge}$.

Proof Setting $\sigma \downarrow \operatorname{CAT}(K)=\operatorname{CAT}(K)$ in the proof of Theorem 4.4(2) shows that ev* is an isomorphism of $p$-adic cohomology, as required.

Remark 4.2 In the special case $X=c(K)$ of Theorem 4.4, a more precise description of $m^{\sigma}(X)$ is possible by appeal to Example 3.1. The action $s_{t_{\sigma, \tau}}: B C^{\sigma} \times B T^{\tau} \rightarrow$ $B T^{\tau}$ is given by $(b, u) \mapsto u t_{\sigma, \tau}(b)$ for every $\tau \supseteq \sigma$, and extends to a morphism $B C^{\sigma} \times B T^{\sigma \downarrow K} \rightarrow B T^{K}$ of diagrams over the inclusion $\sigma \downarrow \operatorname{CAT}(K) \rightarrow \operatorname{CAT}(K)$. Taking colimits and adjointing then creates a map

$$
c\left(\mathrm{st}_{K}(\sigma)\right) \longrightarrow m^{\sigma}(c(K)),
$$


which induces the isomorphism $\theta^{\prime \prime}$ of (24) in mod $p$ cohomology. So its $p$-completion is a homotopy equivalence.

\section{Mapping spaces and $p$-adic cohomology: II}

In this section we prove that the homotopy class of the map $h_{\sigma}$ of Definition 4.3 is uniquely determined, and compute the $p$-adic cohomology of map $\left(B T^{\sigma}, X\right)_{h_{\sigma}}$. We retain the prevailing notation, whereby $X$ is $p$-complete, simply connected, and reflects $K$ over $\mathbb{Z}_{p}^{\wedge}$ by means of an isomorphism $\theta$.

We focus on the action $s_{t_{\sigma}}$ of $B C^{\sigma}$ on $B T^{\sigma}$, given by $(b, u) \mapsto u t_{\sigma}(b)$ in Example 3.1 and denoted here by $s_{\sigma}$. There is a principal $B C^{\sigma}$-fibration

$$
B C^{\sigma} \stackrel{j_{\sigma}}{\longrightarrow} B C^{\sigma} \times B T^{\sigma} \stackrel{s_{\sigma}}{\longrightarrow} B T^{\sigma},
$$

where $j_{\sigma}$ includes the kernel of $s_{\sigma}$ and is given by $b \mapsto\left(b^{-1}, t_{\sigma}(b)\right)$. Any map $g: B T^{\sigma} \rightarrow X$ may be factorised through evaluation at the identity as

$$
B T^{\sigma} \stackrel{g^{\prime}}{\longrightarrow} \operatorname{map}\left(B C^{\sigma}, X\right)_{g \circ t_{\sigma}} \stackrel{\mathrm{ev}}{\longrightarrow} X,
$$

where $g^{\prime}$ is the adjoint of $g \circ s_{\sigma}$, and satisfies $g^{\prime}(u)(c)=g\left(u t_{\sigma}(c)\right)$ for all $u \in B T^{\sigma}$ and $c \in B C^{\sigma}$.

\section{Lemma 5.1 The map}

$$
\operatorname{map}\left(B T^{\sigma}, X\right)_{g} \longrightarrow \operatorname{map}\left(B T^{\sigma}, \operatorname{map}\left(B C^{\sigma}, X\right)_{g \circ t_{\sigma}}\right)_{g^{\prime}}
$$

induced by $s_{\sigma}$ is a homotopy equivalence, whose inverse is induced by evaluation at the identity of $B C^{\sigma}$.

Proof In the context of (25), $g \circ s_{\sigma} \circ j_{\sigma}$ is null-homotopic; it follows from Remarks 3.2(2) that ev: $\operatorname{map}\left(B C^{\sigma}, X\right)_{g \circ s_{\sigma} \circ j_{\sigma}} \rightarrow X$ is a homotopy equivalence. So Zabrodsky's Lemma [18, 3.1] implies that $\circ s_{\sigma}: \operatorname{map}\left(B T^{\sigma}, X\right) \rightarrow \operatorname{map}\left(B C^{\sigma} \times B T^{\sigma}, X\right)_{L}$ is also an equivalence, where $L$ denotes the set of homotopy classes whose composition with $s_{\sigma}$ is trivial. Restricting to the component of $g$ and adjointing yields the required equivalence; by construction, evaluation at the basepoint is a right inverse.

By analogy with (25), $B C^{\sigma}$ also acts on $\operatorname{map}\left(B C^{\sigma}, X\right)_{g \circ t_{\sigma}}$ according to the formula $(b, f) \mapsto f b$, where $f b(c):=f(b c)$.

Lemma 5.2 The map $g^{\prime}: B T^{\sigma} \rightarrow \operatorname{map}\left(B C^{\sigma}, X\right)_{g \circ t_{\sigma}}$ is $B C^{\sigma}$-equivariant. 
Proof For $(b, c) \in B C^{\sigma} \times B C^{\sigma}$, we have $g^{\prime}\left(u t_{\sigma}(b)\right)(c)=g\left(u t_{\sigma}(b) t_{\sigma}(c)\right)$ by (26), and $g^{\prime} b(c)=g\left(u t_{\sigma}(b c)\right)$; these agree, as $t_{\sigma}$ is a homomorphism.

We now insist that $g$ induces the homomorphism $H^{*}\left(h_{\sigma} ; \mathbb{Z}_{p}^{\wedge}\right)$, and write $g=g_{\sigma}$ to emphasise this choice. Thus $g_{\sigma} \circ t_{\sigma} \simeq f_{\sigma}$ by Theorem 3.3(1), and $\operatorname{map}\left(B C^{\sigma}, X\right)_{g \circ t_{\sigma}}$ becomes $m^{\sigma}(X)$. Applying $p$-adic cohomology to (26) shows that $H^{*}\left(g_{\sigma}^{\prime} ; \mathbb{Z}_{p}^{\wedge}\right)=$ $H^{*}\left(h_{\sigma}^{\prime} ; \mathbb{Z}_{p}^{\wedge}\right)$ by Remarks 3.2(1), so we may study our uniqueness problem in terms of maps $B T^{\sigma} \rightarrow m^{\sigma}(X)$, or equivalently, by restricting attention to subcomplexes of the form $\operatorname{st}_{K}(\sigma) \subseteq K$.

We may apply Lemma 4.1 to construct a map $q_{\sigma}: m^{\sigma}(X) \rightarrow\left(B T^{\sigma}\right)_{p}^{\wedge}$ such that $q_{\sigma} \circ g_{\sigma}^{\prime}$ is completion, and consider the homotopy fibration

$$
X_{\ell} \stackrel{i}{\longrightarrow} m^{\sigma}(X) \stackrel{q_{\sigma}}{\longrightarrow}\left(B T^{\sigma}\right)_{p}^{\wedge} .
$$

Combining the Serre spectral sequence for $i$ with Theorem 4.4 shows that $i$ reflects $\left(\operatorname{st}_{K}(\sigma), \ell_{K}(\sigma)\right)$ over $\mathbb{Z}_{p}^{\wedge}$. We take $\theta^{\prime}$ of (23) as the reflector for $m^{\sigma}(X)$, and its quotient by the appropriate ideals as the reflector for $X_{\ell}$.

As a consequence of Lemma 5.2, we may apply the Borel construction to $g_{\sigma}^{\prime}$, and obtain a homotopy pullback diagram of principal $B C^{\sigma}$-fibrations

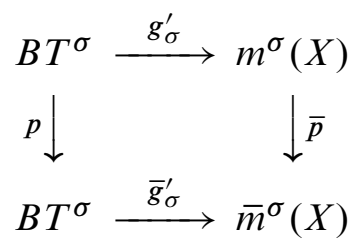

where $\bar{m}^{\sigma}(X):=E B C^{\sigma} \times_{B C} \sigma m^{\sigma}(X)$ and $p$ denotes the $p$-th power homomorphism. So $\bar{m}^{\sigma}(X)$ is simply connected, and applying $\pi_{2}(-)$ to (28) yields the commutative square

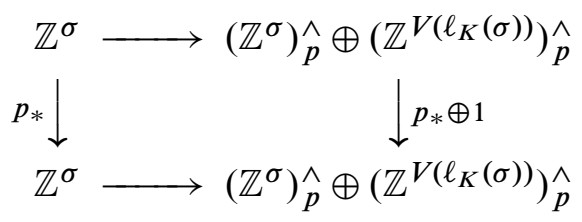

where the horizontal homomorphisms include the completed direct summand $\left(\mathbb{Z}^{\sigma}\right)_{p}^{\wedge}$, and $p_{*}$ is multiplication by $p$.

We now identify $\pi_{2}\left(\bar{m}^{\sigma}(X)\right)$ with $H^{2}\left(\bar{m}^{\sigma}(X) ; \mathbb{Z}_{p}^{\wedge}\right)$ by the universal coefficient theorem, and deduce the existence of a map $\bar{q}_{\sigma}: \bar{m}^{\sigma}(X) \rightarrow\left(B T^{\sigma}\right)_{p}^{\wedge}$ such that $\bar{q}_{\sigma} \circ \bar{g}_{\sigma}^{\prime}$ is completion. The homotopy fibre of $\bar{q}_{\sigma}$ is $X_{\ell}$ because $\bar{q}_{\sigma} \circ \bar{p}$ and $p \circ q_{\sigma}$ are homotopic, and the Serre spectral sequence of the fibration $X_{\ell} \rightarrow \bar{m}^{\sigma}(X) \rightarrow\left(B T^{\sigma}\right)_{p}^{\wedge}$ shows 
that $H^{*}\left(\bar{m}^{\sigma}(X) ; \mathbb{Z}_{p}^{\wedge}\right)$ is of finite type, torsion free, and generated by 2 -dimensional classes.

Lemma 5.3 The space $\bar{m}^{\sigma}(X)$ reflects st $K(\sigma)$ over $\mathbb{Z}_{p}^{\wedge}$; the reflector $\bar{\theta}^{\prime}$ is determined by the commutative square

$$
\begin{array}{cc}
H^{*}\left(\bar{m}^{\sigma}(X) ; \mathbb{Z}_{p}^{\wedge}\right) \stackrel{\bar{\theta}^{\prime}}{\longrightarrow} S_{\mathbb{Z}_{\hat{p}}}(\sigma) \otimes \mathbb{Z}_{p}^{\wedge}\left[\ell_{K}(\sigma)\right] \\
\bar{p}^{*} \downarrow & \downarrow^{p \otimes 1} \\
H^{*}\left(m^{\sigma}(X) ; \mathbb{Z}_{p}^{\wedge}\right) \stackrel{\theta^{\prime}}{\longrightarrow} S_{\mathbb{Z}_{\hat{p}}}(\sigma) \otimes \mathbb{Z}_{p}^{\wedge}\left[\ell_{K}(\sigma)\right]
\end{array}
$$

where $p$ acts on the polynomial generators of $S_{\mathbb{Z}_{\hat{p}}}(\sigma)$ as multiplication by $p$.

Proof The Serre spectral sequence for the $p$-adic cohomology of the fibration $\bar{p}$ of (28) collapses at the $E_{2}$-page, and shows that $\bar{p}^{*}$ is a monomorphism. It is given in dimension 2 by the dual of (29), and the result follows.

At this point it is helpful to work for a while with an arbitrary $p$-complete space $Y$ that reflects $\operatorname{st}_{K}(\sigma)$ over $\mathbb{Z}_{p}^{\wedge}$. We write the reflector as $\theta_{Y}$, on the understanding that it agrees with $\theta^{\prime}$ whenever $Y$ is $m^{\sigma}(X)$; in these circumstances, we also identify $h_{\sigma}: B T^{\sigma} \rightarrow Y$ with $h_{\sigma}^{\prime}: B T^{\sigma} \rightarrow m^{\sigma}(X)$. Of course $h_{\sigma}$ induces the homomorphism $\pi_{\sigma} \circ \theta_{Y}$ in $p$-adic cohomology, where $\pi_{\sigma}$ denotes the projection $S_{\mathbb{Z}_{\hat{p}}}(\sigma) \otimes \mathbb{Z}_{p}^{\wedge}\left[\ell_{K}(\sigma)\right] \rightarrow S_{\mathbb{Z}_{p}}(\sigma)$. We write $g_{\sigma}$ for any other map inducing the same homomorphism, and identify $g_{\sigma}$ with $g_{\sigma}^{\prime}$ in case $Y=m^{\sigma}(X)$.

To analyse $g_{\sigma}$ further, we consider its restriction $g_{\sigma / r}: B C_{r}^{\sigma} \rightarrow Y$, where $C_{r}<T$ denotes the cyclic subgroup of order $p^{r}$ for any $r>0$. We factorise $t_{\sigma}$ into natural inclusions as

$$
B C^{\sigma} \stackrel{\iota_{\sigma / r}}{\longrightarrow} B C_{r}^{\sigma} \stackrel{t_{\sigma / r}}{\longrightarrow} B T^{\sigma},
$$

so that $g_{\sigma / r}=g_{\sigma} \circ t_{\sigma / r}$. The induced homomorphism $H^{*}\left(h_{\sigma / r} ; \mathbb{Z}_{p}^{\wedge}\right)$ is given by $t_{\sigma / r}^{*} \circ \pi_{\sigma} \circ \theta_{Y}$, and Theorem 3.3(1) implies that $g_{\sigma / 1}^{\prime} \simeq h_{\sigma / 1}^{\prime}$ because both are homotopic to $f_{\sigma}$. We abbreviate $\operatorname{map}\left(B C_{r}^{\sigma}, Y\right)_{h_{\sigma / r}}$ to $m_{r}^{\sigma}(Y)$ henceforth.

Proposition 5.4 If $Y$ is $p$-complete and reflects $\mathrm{st}_{K}(\sigma)$ by means of an isomorphism $\theta_{Y}: H^{*}\left(Y ; \mathbb{Z}_{p}^{\wedge}\right) \rightarrow S_{\mathbb{Z}_{\hat{p}}}(\sigma) \otimes \mathbb{Z}_{p}^{\wedge}\left[\ell_{K}(\sigma)\right]$, then

(1) there is a unique homotopy class of maps $h_{\sigma / r}: B C_{r}^{\sigma} \rightarrow Y$ such that $\left(h_{\sigma / r}\right)^{*}=$ $t_{\sigma / r}^{*} \circ \pi_{\sigma} \circ \theta_{Y}$

(2) the map $\circ \iota_{\sigma / r}: m_{r}^{\sigma}(Y) \rightarrow m^{\sigma}(Y)$ is a homotopy equivalence. 
We prove Proposition 5.4 by induction on $r$, following application to our main result. It is convenient to denote $\operatorname{map}\left(B T^{\sigma}, X\right)_{h_{\sigma}}$ by $M^{\sigma}(X)$, and to rewrite the induced map $\circ t_{\sigma}: M^{\sigma}(X) \rightarrow m^{\sigma}(X)$ as $u_{\sigma}$.

Theorem 5.5 If $X$ is $p$-complete and reflects $K$ over $\mathbb{Z}_{p}^{\wedge}$, then

(1) there is a unique homotopy class of maps $h_{\sigma}: B T^{\sigma} \rightarrow X$ that reflect $(K, \Delta(\sigma))$ over $\mathbb{Z}_{p}^{\wedge}$;

(2) the map $u_{\sigma}$ is a homotopy equivalence, and the map ev: $M^{\sigma}(X) \rightarrow X$ reflects $\left(K, \mathrm{st}_{K}(\sigma)\right)$ over $\mathbb{Z}_{p}^{\wedge}$;

(3) there is a map $j: X_{\ell} \rightarrow M^{\sigma}(X)$ that reflects $\left(\operatorname{st}_{K}(\sigma), \ell_{K}(\sigma)\right)$ over $\mathbb{Z}_{p}^{\wedge}$;

(4) there is a homotopy equivalence $M^{\sigma}(X) \simeq\left(B T^{\sigma}\right)_{p}^{\wedge} \times X_{\ell}$.

Proof (1) Write $Y=m^{\sigma}(X)$, and let $l(\sigma)$ be a set of representative maps $B T^{\sigma} \rightarrow Y$ for the homotopy classes that induce $\pi_{\sigma} \circ \theta^{\prime}$ in $p$-adic cohomology. Since $Y$ is $p-$ complete and the natural map $\operatorname{hocolim}_{r} B C_{r}^{\sigma} \rightarrow B T^{\sigma}$ is a mod- $p$ equivalence, the maps

$$
\operatorname{map}\left(B T^{\sigma}, Y\right)_{l(\sigma)} \longrightarrow \operatorname{map}\left(\operatorname{hocolim}_{r} B C_{r}^{\sigma}, Y\right)_{l(\sigma)} \longrightarrow \operatorname{holim}_{r} m_{r}^{\sigma}(Y)
$$

are both equivalences. Furthermore, evaluation at the identity induces compatible equivalences $m_{r}^{\sigma}(Y) \rightarrow Y$ for all $r \geq 1$, by Proposition 5.4. Thus $l(\sigma)$ contains only the homotopy class of $h_{\sigma}^{\prime}$, and (26) implies the result.

(2) The proof of (1) shows that ev $\circ u_{\sigma}: M^{\sigma}(Y) \rightarrow Y$ is also evaluation at the identity, and an equivalence. So Corollary 4.5 confirms that $u_{\sigma}$ is an equivalence. Then apply Lemma 5.1 and Corollary 4.5 to replace $Y$ by $X$ in the source and target respectively; the required reflectors are $\theta_{X}$ for $X$ and $\theta^{\prime} \circ\left(u_{\sigma}^{-1}\right)^{*}$ for $M^{\sigma}(X)$.

(3) By (2), the fibration (27) may be rewritten as

$$
X_{\ell} \stackrel{j}{\longrightarrow} M^{\sigma}(X) \stackrel{r_{\sigma}}{\longrightarrow} B T^{\sigma},
$$

where $i \simeq u_{\sigma} \circ j$ and $r_{\sigma}=q_{\sigma} \circ u_{\sigma}$. The reflectors are those of (2) and (27).

(4) An action $B T^{\sigma} \times M^{\sigma}(X) \rightarrow M^{\sigma}(X)$ is given by $(u, f) \mapsto f u$, where $f u(v)=$ $f(u v)$ for any $u, v \in B T^{\sigma}$ and $f \in M^{\sigma}(X)$. Restricting to $B T^{\sigma} \times X_{\ell}$ along $1 \times j$ induces an isomorphism of $p$-adic cohomology, and is therefore an equivalence.

We now return to the proof of Proposition 5.4; the base cases $r=1$ are immediate, so we assume that $r \geq 2$ throughout. 
Proof (1) Pulling the $p$-th power map on $B T^{\sigma}$ back along $t_{\sigma / r-1}$ and combining the result with (28) and (29) yields a homotopy commutative ladder

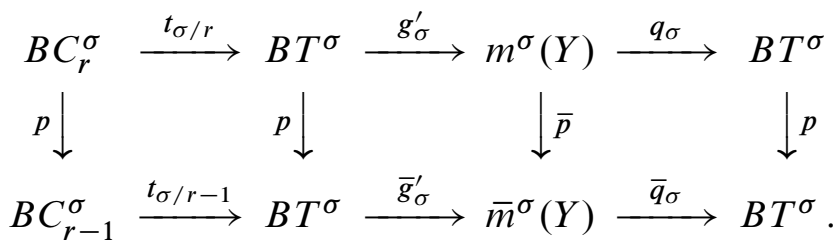

Each vertical map has homotopy fibre $B C^{\sigma}$ and induces a monomorphism in $p$-adic cohomology, so Lemma 5.3 confirms that $H^{*}\left(\bar{g}_{\sigma / r-1}^{\prime} ; \mathbb{Z}_{p}^{\wedge}\right)$ agrees with $H^{*}\left(\bar{h}_{\sigma / r-1}^{\prime} ; \mathbb{Z}_{p}^{\wedge}\right)$ as homomorphisms $H^{*}\left(\bar{m}^{\sigma}(Y) ; \mathbb{Z}_{p}^{\wedge}\right) \rightarrow H^{*}\left(B C_{r-1}^{\sigma} ; \mathbb{Z}_{p}^{\wedge}\right)$. But $\bar{m}^{\sigma}(Y)$ also reflects st $K_{K}(\sigma)$; so the inductive hypotheses show that $\bar{g}_{\sigma / r-1}^{\prime}$ is homotopic to $\bar{h}_{\sigma / r-1}^{\prime}$, and that evaluation

$$
\operatorname{map}\left(B C_{r-1}^{\sigma}, \bar{m}^{\sigma}(Y)\right)_{\bar{h}_{\sigma / r-1}^{\prime}} \stackrel{\circ \iota_{\sigma / r-1}}{\longrightarrow} \operatorname{map}\left(B C^{\sigma}, \bar{m}^{\sigma}(Y)\right)_{\bar{h}_{\sigma / 1}^{\prime}} \stackrel{\text { ev }}{\longrightarrow} \bar{m}^{\sigma}(Y)
$$

at the identity is a homotopy equivalence.

Let $l_{Y}$ denote the set of homotopy classes of lifts $B C_{r}^{\sigma} \rightarrow m^{\sigma}(Y)$ of the map $\bar{p}$ 。 $g_{\sigma / r}^{\prime}: B C_{r}^{\sigma} \rightarrow \bar{m}^{\sigma}(Y)$. Applying the functor map $\left(B C_{r}^{\sigma},-\right)$ to (31) creates a homotopy pullback square

$$
\begin{array}{ccc}
\operatorname{map}\left(B C_{r}^{\sigma}, m^{\sigma}(Y)\right)_{l_{Y}} & \stackrel{q_{\sigma} \circ}{\longrightarrow} & \operatorname{map}\left(B C_{r}^{\sigma}, B T^{\sigma}\right)_{l_{B}} \\
\bar{p} \circ \downarrow & \downarrow p \circ \\
\operatorname{map}\left(B C_{r}^{\sigma}, \bar{m}^{\sigma}(Y)\right)_{\bar{p} \circ g_{\sigma / r}^{\prime}} \stackrel{\bar{q}_{\sigma}^{\circ}}{\longrightarrow} \operatorname{map}\left(B C_{r}^{\sigma}, B T^{\sigma}\right)_{p \circ t_{\sigma / r}}
\end{array}
$$

where $l_{B}$ denotes a set of representatives for homotopy classes of lifts of $p \circ t_{\sigma / r}$. The vertical maps are principal $\operatorname{map}\left(B C_{r}^{\sigma}, B C^{\sigma}\right)$-fibrations by [15], and $\bar{q}_{\sigma} \circ$ may be identified with the corresponding map

$$
\operatorname{map}\left(B C_{r-1}^{\sigma}, \bar{m}^{\sigma}(Y)_{\bar{h}_{\sigma / r-1}^{\prime}} \longrightarrow \operatorname{map}\left(B C_{r-1}^{\sigma}, B T^{\sigma}\right)_{t_{\sigma / r-1} \circ p}\right.
$$

by Zabrodsky's lemma. It follows from the inductive hypothesis that the lower fibration of (33) is equivalent to $\bar{q}_{\sigma}: \bar{m}^{\sigma}(Y) \rightarrow B T^{\sigma}$, and therefore that the common homotopy fibre of the horizontal maps is $Y_{\ell}$, which is 1 -connected. Thus $q_{\sigma} \circ$ induces a bijection $l_{Y} \leftrightarrow l_{B}$ of components. But we are given that $H^{*}\left(g_{\sigma / r}^{\prime} ; \mathbb{Z}_{p}^{\wedge}\right)=H^{*}\left(h_{\sigma / r}^{\prime} ; \mathbb{Z}_{p}^{\wedge}\right)$, from which we deduce that $q_{\sigma} \circ g_{\sigma / r}^{\prime} \simeq q_{\sigma} \circ h_{\sigma / r}^{\prime}$; so $g_{\sigma / r}^{\prime}$ and $h_{\sigma / r}^{\prime}$ define the same element of $l_{Y}$, and are therefore homotopic. Applying ev: $m^{\sigma}(Y) \rightarrow Y$ completes the proof.

(2) The pullback square (33) combines with the inductive hypotheses to show that ev: $\operatorname{map}\left(B C^{\sigma}, m^{\sigma}(Y)\right)_{h_{\sigma / r}^{\prime}} \rightarrow m^{\sigma}(Y)$ is an equivalence, and Corollary 4.5 allows us 
to replace $m^{\sigma}(Y)$ with $Y$, and $h_{\sigma / r}^{\prime}$ with $h_{\sigma / r}$. Then $\circ \iota_{\sigma / r}$ is also an equivalence, by analogy with (32).

\section{Higher limits of diagrams}

In this section we recall the basic properties of higher limits of diagrams $\Phi: \mathrm{C} \rightarrow \mathrm{AB}$ of abelian groups, where $\mathrm{C}$ is usually of the form $\mathrm{CAT}^{\mathrm{op}}(K)$. These are crucial for later sections, and also featured in [11, Section 3].

Following Oliver's elaboration [12] of [2], we interpret $\lim _{\mathrm{C}}^{i} \Phi$ as the $i$-th cohomology group of the cochain complex $\left(C^{*}(\mathrm{C}, \Phi), \delta\right)$, where

$$
C^{n}(\mathrm{C} ; \Phi)=\prod_{c_{0} \rightarrow c_{1} \rightarrow \ldots \rightarrow c_{n}} \Phi\left(c_{n}\right)
$$

for every $n \geq 0$, and the product is taken over all chains of morphisms in $\mathrm{C}$. The differential $\delta: C^{n}(\mathrm{C} ; \Phi) \rightarrow C^{n+1}(\mathrm{C} ; \Phi)$ is the alternating sum $\sum_{k=0}^{r}(-1)^{k} \delta^{k}$, where $\delta^{k}$ is defined on $u \in C^{n}(\mathrm{C} ; \Phi)$ by

$$
\delta^{k}(u)\left(c_{0} \rightarrow \cdots \rightarrow c_{n+1}\right):= \begin{cases}u\left(c_{0} \rightarrow \cdots \rightarrow \widehat{c_{k}} \rightarrow \cdots \rightarrow c_{n+1}\right) & \text { for } k \leq n, \\ \Phi\left(c_{n} \rightarrow c_{n+1}\right) u\left(c_{0} \rightarrow \cdots \rightarrow c_{n}\right) & \text { for } k=n+1 .\end{cases}
$$

We may replace $C^{*}(\mathrm{C} ; \Phi)$ by its quotient $N^{*}(\mathrm{C} ; \Phi)$ of normalised cochains, obtained by restricting the products (34) to chains of nonidentities. So

$$
\lim _{\mathrm{C}}^{i} \Phi:=H^{i}\left(C^{*}(\mathrm{C} ; \Phi), \delta\right) \cong H^{i}\left(N^{*}(\mathrm{C} ; \Phi), \delta\right)
$$

for $i \geq 0$, and the limits are themselves abelian groups. The same construction works for small diagrams in an arbitrary abelian category A, and the limits inherit any additional algebraic structure from the objects of $\mathrm{A}$.

Given a constant diagram $\operatorname{cst}_{M}: \mathrm{C} \rightarrow \mathrm{AB}$, it follows that its higher limits are determined by the cohomology of the classifying space $B \mathrm{C}$, via an isomorphism $\lim _{\mathrm{C}}^{i} \operatorname{cst}_{M} \cong$ $H^{i}(B \mathrm{C} ; M)$. In particular, $\lim _{\mathrm{CAT}}^{i}(K) \operatorname{cst}_{M}$ vanishes for $i \geq 1$ because $B \operatorname{CAT}^{\mathrm{op}}(K)$ is contractible, and $\lim _{\mathrm{CAT}}^{i}\left(K^{\times}\right) \operatorname{cst}_{M}$ is isomorphic to $H^{i}(K ; M)$ because $B \operatorname{CAT}^{\mathrm{op}}\left(K^{\times}\right)$ is homeomorphic to the realisation $|K|$. Both are isomorphic to $M$ when $i=0$.

For any face $\sigma \in K$, we refer to a diagram $\Phi: \mathrm{CAT}^{\mathrm{op}}(K) \rightarrow \mathrm{AB}$ as $\sigma$-atomic when $\Phi(\tau)=0$ for every $\tau \neq \sigma$. We consider the following generalisation of [11, Lemma 3.9], in which it is convenient to set $\tilde{H}^{n}(\varnothing ; M):=0$ for $n \geq 0$, and $\tilde{H}^{-1}(\varnothing ; M):=M$, for any abelian group $M$. 
Proposition 6.1 If $\Phi$ is $\sigma$-atomic, then there exists an isomorphism

$$
\lim _{\mathrm{CAT}^{\mathrm{op}}(K)}^{i} \Phi \cong \tilde{H}^{i-1}\left(\ell_{K}(\sigma) ; \Phi(\sigma)\right)
$$

for any $i \geq 0$.

Proof Let $J=\ell_{K}(\sigma)$, and define $\Phi^{\prime}: \mathrm{CAT}^{\mathrm{op}}(J) \rightarrow \mathrm{AB}$ by $\Phi^{\prime}(\tau \backslash \sigma):=\Phi(\tau)$ for every $\tau \supseteq \sigma$. Thus $\Phi^{\prime}$ is $\varnothing$-atomic, and $\Phi^{\prime} \circ P^{\mathrm{op}}=\left.\Phi\right|_{\mathrm{CAT}^{\mathrm{op}}(K) \downarrow \sigma}$ in terms of the functor $P$ of (5). The isomorphisms

$$
N^{*}\left(\mathrm{CAT}^{\mathrm{op}}(K) ; \Phi\right) \cong N^{*}\left(\mathrm{CAT}^{\mathrm{op}}(K) \downarrow \sigma ; \Phi\right) \cong N^{*}\left(\mathrm{CAT}^{\mathrm{op}}(J) ; \Phi^{\prime}\right)
$$

of normalised chain complexes determine an isomorphism

$$
\lim _{\mathrm{CAT} \mathrm{op}(K)}^{i} \Phi \cong \lim _{\mathrm{CAT} \mathrm{op}(J)}^{i} \Phi^{\prime}
$$

for every $i \geq 0$.

If $J=\{\varnothing\}$, the claim is clear. Otherwise, write $\Phi^{\prime}(\varnothing)$ as $M$ and consider the short exact sequence

$$
0 \longrightarrow \Phi^{\prime} \longrightarrow \operatorname{cst}_{M} \longrightarrow \Upsilon \longrightarrow 0
$$

of $\operatorname{CAT}^{\mathrm{op}}(J)$-diagrams, where $\Upsilon$ is trivial on $\varnothing$ and constant on the subcategory $\operatorname{CAT}^{\mathrm{op}}\left(J^{\times}\right)$. The long exact sequence associated to (35) combines with the isomorphism $N^{*}\left(\mathrm{CAT}^{\mathrm{op}}(J) ; \Upsilon\right) \cong N^{*}\left(\mathrm{CAT}^{\mathrm{op}}\left(J^{\times}\right) ; \operatorname{cst}_{M}\right)$ to ensure that

$$
\lim _{\mathrm{CAT} T^{\mathrm{op}}(J)}^{i} \Phi^{\prime} \cong \lim _{\mathrm{CAT} T^{\mathrm{OP}}(J)}^{i-1} \Upsilon \cong \widetilde{H}^{i-1}(J ; M)
$$

for $i \geq 2$, and reduces to the short exact sequence

$$
0 \longrightarrow M \longrightarrow H^{0}(J ; M) \longrightarrow \lim ^{1} \Phi^{\prime} \longrightarrow 0
$$

for $i=1$. So the result holds for all $i$.

We may associate a $\sigma$-atomic diagram $\Phi_{\sigma}$ to every $\Phi$, by the formula

$$
\Phi_{\sigma}(\tau)= \begin{cases}\Phi(\sigma) & \text { when } \tau=\sigma \\ 0 & \text { otherwise }\end{cases}
$$

Such diagrams play a fundamental rôle in proving vanishing results for higher limits; in particular, they act as building blocks for the following notions of filtration, which depend on the poset structure of $\mathrm{CAT}^{\mathrm{op}}(K)$.

Given $\Phi$ as above, $\Phi_{s}, \Phi_{\geq s}: \mathrm{CAT}^{\mathrm{op}}(K) \rightarrow \mathrm{AB}$ are defined by

$$
\Phi_{s}(\sigma)=\left\{\begin{array}{ll}
\Phi(\sigma) & \text { if }|\sigma|=s, \\
0 & \text { otherwise, }
\end{array} \quad \text { and } \quad \Phi_{\geq s}(\sigma)= \begin{cases}\Phi(\sigma) & \text { if }|\sigma| \geq s \\
0 & \text { otherwise }\end{cases}\right.
$$


Thus $\Phi_{s}$ decomposes as the product diagram $\prod_{|\sigma|=s} \Phi_{\sigma}$. Also, $\Phi_{\geq 0}=\Phi$ and $\Phi_{\geq \operatorname{dim} K+1}=\Phi_{\operatorname{dim} K+1}$ hold by definition, and $\Phi_{\geq \operatorname{dim} K+2}$ is trivial. Furthermore, there is a short exact sequence

$$
0 \longrightarrow \Phi_{s} \longrightarrow \Phi_{\geq s} \longrightarrow \Phi_{\geq s+1} \longrightarrow 0
$$

for every $s>0$, because there are no morphisms $\sigma \rightarrow \tau$ when $|\tau|<|\sigma|$.

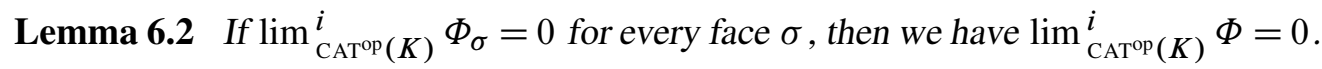

Proof The decomposition of $\Phi_{S}$ into atomic factors ensures that $\lim _{\mathrm{CAT}(K)}^{i} \Phi_{S}$ is zero, and the long exact sequence associated to (36) confirms that

$$
\lim _{\mathrm{CAT}^{\mathrm{op}}(K)}^{i} \Phi_{\geq s} \longrightarrow \lim _{\mathrm{CAT}^{\mathrm{op}}(K)}^{i} \Phi_{\geq s+1}
$$

is a monomorphism for every $0 \leq s \leq \operatorname{dim} K+1$. So the composition

$$
\lim _{\mathrm{CAT}(K)}^{i} \Phi \longrightarrow \lim _{\mathrm{CAT}(K)}^{i} \Phi_{\geq \operatorname{dim} K+2}=0
$$

is also a monomorphism, and the result follows.

Lemma 6.3 If $i \geq \operatorname{dim} K+2$, then we have that $\lim _{\mathrm{CAT}(K)}^{i} \Phi=0$.

Proof The cochain group $N^{i}\left(\mathrm{CAT}^{\mathrm{op}}(K) ; \Psi\right)$ vanishes for $i \geq \operatorname{dim} K+2$.

Given any two diagrams $\Phi: \mathrm{CAT}^{\mathrm{op}}(K) \rightarrow \mathrm{AB}$ and $\Psi: \mathrm{CAT}^{\mathrm{op}}(L) \rightarrow \mathrm{AB}$, we define their external product $\Phi \times \Psi: \mathrm{CAT}^{\mathrm{op}}(K * L) \rightarrow \mathrm{AB}$ by $\Phi \times \Psi(\sigma \cup \tau):=\Phi(\sigma) \times \Psi(\tau)$.

Lemma 6.4 For any $i \geq 1$, there is an isomorphism

$$
\lim _{\mathrm{CAT} T^{\mathrm{op}}(K * L)}^{i} \Phi \times \Psi \cong \lim _{\mathrm{CAT}(K)}^{i} \Phi \times \lim _{\mathrm{CAT}(L)}^{i} \Psi .
$$

Proof This follows directly from the isomorphism

$$
C^{*}\left(\mathrm{CAT}^{\mathrm{op}}(K * L) ; \Phi \times \Psi\right) \cong C^{*}\left(\mathrm{CAT}^{\mathrm{op}}(K) ; \Phi\right) \times C^{*}\left(\mathrm{CAT}^{\mathrm{op}}(L) ; \Psi\right)
$$

of cochain complexes. 


\section{$7 \quad p$-adic homotopy uniqueness for $\Delta^{(r)}(V)$}

In this section we show that the homotopy type of the $p$-adic completion $\operatorname{hc}(K)_{p}^{\wedge}$ is determined by its $p$-adic cohomology, for any $r$-skeleton $\Delta^{(r)}(V)$ of the $(m-1)-$ simplex. We restrict attention to 1 -connected spaces, so that completion is well-behaved. We also maintain the notation of previous sections, but make the additional assumption that any space denoted by $X$ or $X_{J}$ is $p$-complete, for every simplicial complex $J$.

Our aim is to prove that $K=\Delta^{(r)}(V)$ satisfies the following.

\section{Conditions 7.1}

(C1) There is an isomorphism $\theta: H^{*}\left(X ; \mathbb{Z}_{p}^{\wedge}\right) \rightarrow \mathbb{Z}_{p}^{\wedge}[K]$ if and only if there is a homotopy equivalence $f: \operatorname{hc}(K)_{p}^{\wedge} \rightarrow X$ such that $f^{*}=\theta$.

(C2) Any two self-equivalences $f, g: \operatorname{hc}(K)_{p}^{\wedge} \rightarrow \mathrm{hc}(K)_{p}^{\wedge}$ are homotopic if and only if $H^{*}\left(f ; \mathbb{Z}_{p}^{\wedge}\right)=H^{*}\left(g ; \mathbb{Z}_{p}^{\wedge}\right)$.

To confirm (C1), it suffices to assume the existence of $\theta$ and construct a mod $p$ equivalence $f: \operatorname{hc}(K) \rightarrow X$ such that $f^{*}=\theta$. Our strategy is to apply the results of Section 4 and Section 5.

By Corollary 4.2, there are maps $h_{\sigma}: B T^{\sigma} \rightarrow X$ for all faces $\sigma \in K$, which induce the restrictions of $\theta$ in $p$-adic cohomology. By Theorem 5.5(1), the $h_{\sigma}$ are compatible up to homotopy with the inclusions $B T^{\sigma} \rightarrow B T^{\tau}$ for all $\sigma \subseteq \tau$. They therefore define a map $(\operatorname{hc}(K))^{(1)} \rightarrow X$ on the 1 -skeleton of the homotopy colimit hc $(K)$. The Bousfield-Kan spectral sequence for homotopy inverse limits, together with work of Wojtkowiak [17] clarifying the situation for low dimensions, provides an obstruction theory for extending this map to $\mathrm{hc}(K)$. The obstruction groups are given by the higher limits

$$
\lim _{\mathrm{CAT} \text { op }(K)}^{i+1} \pi_{i}\left(M^{\sigma}(X)\right) .
$$

If these groups vanish for all $i \geq 1$, then the desired extension exists. So for any $i \geq 1$, it is convenient to define the diagram

$$
\Pi_{i}: \mathrm{CAT}^{\mathrm{op}}(K) \longrightarrow \mathrm{AB}
$$

by assigning $\pi_{i}\left(M^{\sigma}(X)\right)$ to each face $\sigma$, and assigning the homomorphism induced by $M^{\tau}(X) \rightarrow M^{\sigma}(X)$ to each morphism $\tau \supseteq \sigma$.

The obstruction theory also applies to the question of uniqueness. There is a restriction map

$$
R:[\mathrm{hc}(K), X] \longrightarrow \lim _{\mathrm{CAT}^{\mathrm{op}}(K)}^{0}\left[B T^{K}, X\right]
$$


of homotopy classes, whose target may be identified with $\left[\mathrm{hc}(K)^{(1)}, X\right]$. If, for some element $h_{1}:=\left(h_{\sigma}\right)_{\sigma \in K}$ of the latter, the limits $\lim _{\mathrm{CAT}}^{i}(K) \Pi_{i}$ vanish for all $i \geq 1$, then $R^{-1}\left(h_{1}\right)$ contains at most one element; in other words, there is at most one extension hc $(K) \rightarrow X$ of $h_{1}$, up to homotopy.

So we are interested in simplicial complexes $K$ that satisfy the following.

Condition 7.2 (VHL) For any $p$-complete space $X$ equipped with an isomorphism $\theta: H^{*}\left(X ; \mathbb{Z}_{p}^{\wedge}\right) \rightarrow \mathbb{Z}_{p}^{\wedge}[K]$, the groups

$$
\lim _{\mathrm{CAT} \mathrm{OP}(K)}^{i} \Pi_{i} \quad \text { and } \quad \lim _{\mathrm{CAT}(K)}^{i+1} \Pi_{i}
$$

vanish for all $i \geq 1$.

Proposition 7.3 If $K$ satisfies (VHL), then it satisfies (C1) and (C2).

Proof If $K$ satisfies (VHL), the above considerations show that there exists a map $f: \operatorname{hc}(K) \rightarrow X$ which induces $\theta$. It therefore determines the homotopy equivalence $f: \operatorname{hc}(K)_{p}^{\wedge} \rightarrow X$ required for $(\mathrm{C} 1)$.

Let $f, g: \operatorname{hc}(K)_{p}^{\wedge} \rightarrow \operatorname{hc}(K)_{p}^{\wedge}$ be self-equivalences, and $H^{*}\left(f ; \mathbb{Z}_{p}^{\wedge}\right)=H^{*}\left(g ; \mathbb{Z}_{p}^{\wedge}\right)$. Without loss of generality, we may assume that $f$ is the identity. Then for every face $\sigma \in K$, the restriction $\left.g\right|_{B T^{\sigma}}$ reflects the pair $(K, \Delta(\sigma))$ over $\mathbb{Z}_{p}^{\wedge}$, and so by Theorem $5.5(1)$ is homotopic to $h_{\sigma}$; in particular, $g$ lies in $R^{-1}\left(\left(h_{\sigma}\right)_{\sigma \in K}\right)$. All obstruction groups vanish by assumption, so 1 and $g$ are homotopic, as required for (C2).

We now turn to the distinguishing properties of the skeleta $\Delta^{(r)}(V)$. For any space $X$ that reflects $K$ over $\mathbb{Z}_{p}^{\wedge}$, we denote the homotopy fibre of the map $q_{X}: X \rightarrow B T^{V}$ of Lemma 4.1 by $F_{X}$.

Lemma 7.4 For $K=\Delta^{(r-1)}(V)$ and any face $\sigma$, we have that

(1) the space $F_{X}$ is $2 r$-connected;

(2) $\ell_{K}(\sigma)$ is empty if $\sigma$ is maximal, and is $\Delta^{(r-|\sigma|-1)}(V \backslash \sigma)$ otherwise.

Proof (1) The missing faces of $K$ have cardinality at least $r+1$, so $q_{X}$ induces an isomorphism of $p$-adic cohomology in dimensions $\leq 2 r+1$. The same is therefore true in homology. Both $X$ and $B T^{V}$ are simply connected and $p$-complete, so $\pi_{j}\left(F_{X}\right)=0$ for $1 \leq j \leq 2 r$ by Whitehead's Theorem.

(2) The faces $\rho$ of $\ell_{K}(\sigma)$ are restricted only by the requirements that they are subsets of $V \backslash \sigma$, and that $|\sigma|+|\rho| \leq r$. 
Lemma 7.4(2) combines with (9) and Theorem 5.5(3)-(4) to create a homotopy pullback square

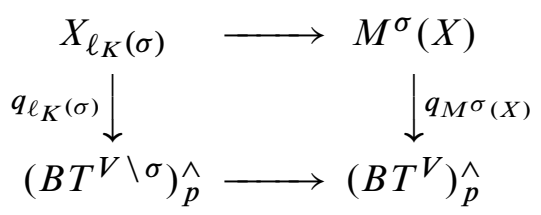

for every nonmaximal face $\sigma \in K$; when $\sigma$ is maximal, there is an equivalence $M^{\sigma}(X) \simeq\left(B T^{\sigma}\right)_{p}^{\wedge}$.

Theorem 7.5 Suppose that $K=\Delta^{(r)}(V)$, and $X$ reflects $K$ over $\mathbb{Z}_{p}^{\wedge}$ : then we have that $\lim _{\mathrm{CAT}^{\mathrm{op}}(K)}^{j} \Pi_{i}=0$ for all $j \geq i$.

Proof If $i=1$, then $\Pi_{1}(\sigma)=0$ for every face $\sigma$, by Theorem 5.5(2); therefore $\lim _{\mathrm{CAT} \text { op }(K)}^{j} \Pi_{1}=0$ for $j \geq 1$.

If $i=2$, then $\Pi_{2}(\sigma)=\left(\mathbb{Z}^{\sigma}\right)_{p}^{\wedge}$ when $\sigma$ is maximal, and $\left(\mathbb{Z}^{V}\right)_{p}^{\wedge}$ otherwise, by (37). So there exists a short exact sequence

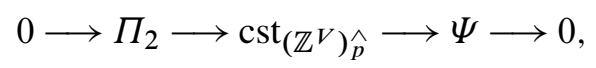

where $\Psi$ is nonzero only on maximal faces, and decomposes as a product of atomic

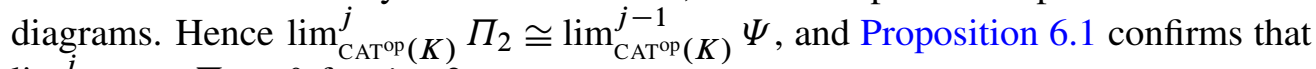
$\lim _{\mathrm{CAT}(K)}^{j} \Pi_{2}=0$ for $j \geq 2$.

If $i \geq 3$, abbreviate the atomic diagrams $\left(\Pi_{i}\right)_{\sigma}$ to $\Pi_{\sigma}$, and the groups $\lim _{\mathrm{CAT}}^{j}(K) \Pi_{\sigma}$ to $l_{\sigma}^{j}$. It follows from Lemma 7.4(1) and (37) that $\Pi_{\sigma}=0$ for all $\sigma$ satisfying $|\sigma| \leq r-i / 2$, because the fibre of $q_{\ell_{K}(\sigma)}$ is $2(r-|\sigma|)$-connected. So $l_{\sigma}^{j}=0$ for $j \geq i$. The remaining faces satisfy $i>2(r-|\sigma|)$, and therefore $i>(r-|\sigma|)$. But Proposition 6.1 and Lemma 7.4(2) identify $l_{\sigma}^{j}$ with $\widetilde{H}^{j-1}\left(\Delta^{(r-|\sigma|-1)}(V \backslash \sigma) ; \Phi(\sigma)\right)$. Hence $l_{\sigma}^{j}=0$ for $j \geq i$ in these cases also, and the result follows by applying Lemma 6.2.

Theorem 7.5 shows that $K$ satisfies (VHL), so the following is an immediate consequence of Proposition 7.3.

Corollary 7.6 Every complex $\Delta^{(r)}(V)$ satisfies conditions $(\mathrm{C} 1)$ and $(\mathrm{C} 2)$.

For low dimensional $K$, our main results hold without restriction.

Theorem 7.7 (1) For $\operatorname{dim}(K) \leq 1$, there is an isomorphism $\theta: H^{*}\left(X ; \mathbb{Z}_{p}^{\wedge}\right) \rightarrow$ $\mathbb{Z}_{p}^{\wedge}[K]$ if and only if there is a homotopy equivalence $f: \operatorname{hc}(K)_{p}^{\wedge} \rightarrow X$ such that $f^{*}=\theta$.

(2) For $\operatorname{dim}(K)=0$, two self equivalences $f, g: \mathrm{hc}(K)_{p}^{\wedge} \rightarrow \mathrm{hc}(K)_{p}^{\wedge}$ are homotopic if and only if $H^{*}\left(f ; \mathbb{Z}_{p}^{\wedge}\right)=H^{*}\left(g ; \mathbb{Z}_{p}^{\wedge}\right)$. 
Proof All relevant mapping spaces are simply connected, so (1) follows from the fact that $\lim ^{j} \Pi_{i}(\sigma)$ vanishes for dimensional reasons when $j \geq 3$. If $\operatorname{dim}(K)=0$ then $K=\Delta^{(0)}(V)$ for some $V$; so (2) follows from Corollary 7.6.

\section{Joins of complexes and higher limits}

We have shown in Section 7 that skeleta of simplices satisfy condition (VHL), and the aim of Proposition 8.5 is to extend the class of complexes with this property. Preparing for the proof occupies most of this section, throughout which we insist that $X=X_{K * L}$ reflects $K * L$ over $\mathbb{Z}_{p}^{\wedge}$. We maintain our convention that any space denoted by $X$ or $X_{J}$ is $p$-complete, for every simplicial complex $J$.

Lemma 8.1 For any $i_{K}$ reflecting $(K * L, K)$ and $i_{L}$ reflecting $(K * L, L)$ over $\mathbb{Z}_{p}^{\wedge}$, the homotopy pullback of the diagram

$$
X_{K} \stackrel{i_{K}}{\longrightarrow} X \stackrel{i_{L}}{\longleftarrow} X_{L}
$$

is $p$-complete and contractible.

Proof The pullback is $p$-complete by [2, Section II.5], and the Eilenberg-Moore spectral sequence computes its mod $p$ cohomology. The $E_{2}$-term is given by

$$
\operatorname{Tor}_{\mathbb{F}_{p}[K] \otimes \mathbb{F}_{p}[L]}^{j}\left(\mathbb{F}_{p}[K], \mathbb{F}_{p}[L]\right)= \begin{cases}0 & \text { for } j \neq 0, \\ \mathbb{F}_{p} & \text { for } j=0,\end{cases}
$$

so the spectral sequence collapses and the pullback is contractible.

The homotopy fibre of $i_{L}$ is therefore the loop space $\Omega X_{K}$, and its inclusion into $X_{L}$ is null-homotopic.

Lemma 8.2 For any $i_{L}$ reflecting $(K * L, L)$ over $\mathbb{Z}_{p}^{\wedge}$, and any face $\tau \in L$, the square

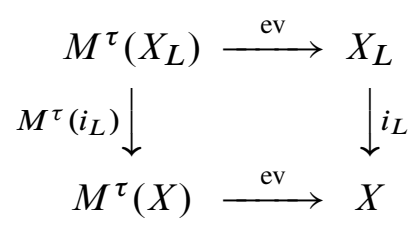

is a homotopy pullback and functorial in $\tau$. 
Proof First apply the functor $\operatorname{map}\left(B T^{\tau},-\right)$ to $i_{L}$, and use evaluation at the base point to obtain a commutative square whose upper left-hand entry is $M^{\tau}\left(X_{L}\right)$. By Theorems 4.2 and 5.5(2), the upper evaluation reflects $\left(L, \mathrm{st}_{L}(\tau)\right)$ over $\mathbb{Z}_{p}^{\wedge}$; and there is a canonical map $d: M^{\tau}\left(X_{L}\right) \rightarrow P$ to the pullback $P$.

The Eilenberg-Moore spectral sequence computes $H^{*}\left(P ; \mathbb{F}_{p}\right)$. The isomorphisms

$$
\operatorname{Tor}_{\mathbb{F}_{p}[K] \otimes \mathbb{F}_{p}[L]}^{j}\left(\mathbb{F}_{p}[K] \otimes \mathbb{F}_{p}\left[\operatorname{st}_{L}(\tau)\right], \mathbb{F}_{p}[L]\right) \cong \operatorname{Tor}_{\mathbb{F}_{p}}^{j}\left(\mathbb{F}_{p}\left[\operatorname{st}_{L}(\tau)\right], \mathbb{F}_{p}\right)
$$

show that the higher Tor groups vanish, and that the spectral sequence collapses at the $E_{2}$-page. It follows that $d$ is a homotopy equivalence, as required. Functoriality of the construction is immediate.

Lemma 8.3 Suppose that $L$ satisfies condition (VHL); then there exists a map $j_{L}: \operatorname{hc}(L) \rightarrow X$ reflecting $(K * L, L)$ over $\mathbb{Z}_{p}^{\wedge}$, and it is unique up to homotopy.

Proof Consider a maximal face $\mu$ of the subcomplex $K \subseteq K * L$, so that $\ell_{K * L}(\mu)=L$. The fibre inclusion $X_{L} \rightarrow M^{\mu}(X)$ of Theorem 5.5(3) may be combined with the equivalence $\mathrm{hc}(L) \rightarrow X_{L}$ that arises because $L$ satisfies (VHL). Then composition with evaluation at the basepoint defines $j_{L}$ as hc $(L) \rightarrow M^{\mu}(X) \rightarrow X$, which reflects $(K * L, K)$ over $\mathbb{Z}_{p}^{\wedge}$ by Theorem 5.5.

In order to prove homotopy uniqueness, we utilise the obstruction theory of Section 7. In this case the obstruction groups are $\lim _{\mathrm{CAT}}^{i}(L) \pi_{i}\left(M^{\tau}(X)\right)$ for $i \geq 1$, and it remains to show that they vanish.

First apply Lemma 8.1 and Lemma 8.2 to the maps $j_{K}$ and $j_{L}$. The homotopy exact sequences of the principal fibrations $\Omega \mathrm{hc}(K) \rightarrow M^{\tau}(\mathrm{hc}(L)) \rightarrow M^{\tau}(X)$ decompose into short exact sequences

$$
0 \longrightarrow \pi_{i}\left(M^{\tau}(\mathrm{hc}(L))\right) \longrightarrow \pi_{i}\left(M^{\tau}(X)\right) \longrightarrow \operatorname{cst}_{\pi_{i}(\mathrm{hc}(K))} \longrightarrow 0
$$

of $\mathrm{CAT}^{\mathrm{op}}(L)$-diagrams. But $L$ satisfies (VHL) by assumption, and the higher limits of constant diagrams are zero on $\mathrm{CAT}^{\mathrm{op}}(L)$. So (38) implies $\lim _{\mathrm{CAT}}^{i}(L) \pi_{i}\left(M^{\tau}(X)\right)=0$ for $i \geq 1$, as required.

Lemma 8.4 Suppose that $K$ and $L$ satisfy (VHL); then for any $\sigma \in K$ and $\tau \in L$ there exist maps $l_{K}(\sigma, \tau)$ reflecting $\left(\operatorname{st}_{K}(\sigma) * \mathrm{st}_{L}(\tau)\right.$, st $\left.K_{K}(\sigma)\right)$ and $l_{L}(\tau, \sigma)$ reflecting $\left(\mathrm{st}_{K}(\sigma) * \mathrm{st}_{L}(\tau), \mathrm{st}_{L}(\tau)\right)$ over $\mathbb{Z}_{p}^{\wedge}$, such that the diagram

$$
M^{\sigma}(\mathrm{hc}(K)) \stackrel{l_{K}(\sigma, \tau)}{\longrightarrow} M^{\sigma \cup \tau}(X) \stackrel{l_{L}(\tau, \sigma)}{\longleftarrow} M^{\tau}(\mathrm{hc}(L))
$$

is homotopy functorial in $\sigma$ and $\tau$ and has contractible homotopy pullback. 
Proof In order to define $l_{K}(\sigma, \tau)$ and $l_{L}(\tau, \sigma)$, observe that the respective restrictions of $h_{\sigma \cup \tau}: B T^{\sigma \cup \tau} \rightarrow X$ to $B T^{\sigma}$ and $B T^{\tau}$ are homotopic to $h_{\sigma}$ and $h_{\tau}$, by Theorem 5.5(1). So the adjoint properties of mapping spaces provide homeomorphisms

$$
M^{\tau}\left(M^{\sigma}(X)\right) \equiv M^{\sigma \cup \tau}(X) \equiv M^{\sigma}\left(M^{\tau}(X)\right) .
$$

By Theorem 5.5(2) and Lemma 8.3, there exists $j_{L}(\sigma)$ : hc $(L) \rightarrow M^{\sigma}(X)$ reflecting $\left(\operatorname{st}_{K}(\sigma) * L, L\right)$ over $\mathbb{Z}_{p}^{\wedge}$. Then $l_{L}(\tau, \sigma)$ is induced by applying $\operatorname{map}\left(B T^{\tau},-\right)$ to $j_{L}(\sigma)$, and reflects $\left(\operatorname{st}_{K}(\sigma) * \mathrm{st}_{L}(\tau), \mathrm{st}_{L}(\tau)\right)$ over $\mathbb{Z}_{p}^{\wedge}$ by Theorem 5.5(2). The construction is functorial in $\tau$, and homotopy functorial in $\sigma$ because Lemma 8.3 defines $j_{L}(\sigma)$ uniquely up to homotopy. Interchanging $\sigma \in K$ and $\tau \in L$ defines $l_{K}(\sigma, \tau)$, which reflects $\left(\operatorname{st}_{K}(\sigma) * \mathrm{st}_{L}(\tau)\right.$, st $\left.\mathrm{st}_{K}(\sigma)\right)$ over $\mathbb{Z}_{p}^{\wedge}$; the construction is functorial in $\sigma$ and homotopy functorial in $\tau$.

The homotopy pullback of the diagram is contractible by Lemma 8.1 .

Proposition 8.5 The class of simplicial complexes satisfying (VHL) is closed under the formation of finite joins.

Proof Let $K$ and $L$ satisfy (VHL). By Lemma 8.4, there are isomorphisms

$$
\pi_{i}\left(M^{\sigma}(\mathrm{hc}(K))\right) \times \pi_{i}\left(M^{\tau}(\mathrm{hc}(L))\right) \cong \pi_{i}\left(M^{\tau \cup \sigma}(X)\right)
$$

that are functorial on $\mathrm{CAT}^{\mathrm{op}}(K) \times \mathrm{CAT}^{\mathrm{op}}(L)$, and so on $\mathrm{CAT}^{\mathrm{op}}(K * L)$. The result follows from Lemma 6.4, by induction on the number of factors.

We believe that other combinatorial operations on simplicial complexes may also respect condition (VHL).

Theorem 8.6 Let $X$ be a $p$-complete $C W$-complex, and suppose that $K$ is an iterated join $\Delta^{\left(r_{1}\right)}\left(U_{1}\right) * \cdots * \Delta^{\left(r_{t}\right)}\left(U_{t}\right)$ of skeleta of simplices; then there is an isomorphism $\theta: H^{*}\left(X ; \mathbb{Z}_{p}^{\wedge}\right) \rightarrow \mathbb{Z}_{p}^{\wedge}[K]$ if and only if there is a homotopy equivalence $f: \operatorname{hc}(K)_{p}^{\wedge} \rightarrow X$ such that $f^{*}=\theta$.

Proof Any such $K$ satisfies condition (VHL) by Theorem 7.5 and Proposition 8.5, and therefore satisfies $(\mathrm{C} 1)$ by Proposition 7.3.

Theorem 8.7 For complexes $K$ as in Theorem 8.6, any pair of self-equivalences $f, g: \operatorname{hc}(K)_{p}^{\wedge} \rightarrow \operatorname{hc}(K)_{p}^{\wedge}$ are homotopic if and only if $H^{*}\left(f ; \mathbb{Z}_{p}^{\wedge}\right)=H^{*}\left(g ; \mathbb{Z}_{p}^{\wedge}\right)$.

Proof As before, $K$ satisfies (C2) by Proposition 7.3. 


\section{The integral homotopy type}

Our final aim is to prove Theorems 9.4 and 9.5. For any space $Y$, we denote the product of all its $p$-adic completions by $Y^{\wedge}:=\prod_{p} Y_{p}^{\wedge}$, and its rationalisation by $Y_{0}$; its finite adele type $Y_{\mathbb{A}_{f}}$ is both the rationalisation of $Y^{\wedge}$ and the formal completion of $Y_{0}$. When $Y$ is nilpotent, these spaces fit into Sullivan's arithmetic pullback square

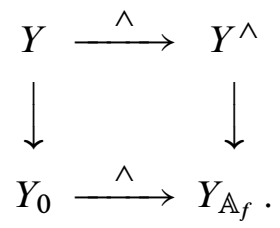

Given any partition $\mathcal{U}:=\left\{U_{0}, \ldots, U_{t}\right\}$ of $V$, we write $K(\mathcal{U})$ for the iterated join $\Delta\left(U_{0}\right) * \partial\left(U_{1}\right) * \cdots * \partial\left(U_{t}\right)$. So there is an isomorphism

$$
\mathbb{Q}\left[K(\mathcal{U}] \cong \mathbb{Q}\left[\Delta\left(U_{0}\right)\right] \otimes \mathbb{Q}\left[\partial\left(U_{1}\right)\right] \otimes \cdots \otimes \mathbb{Q}\left[\partial\left(U_{t}\right)\right],\right.
$$

which implies the following observation of [11, Section 5].

Proposition 9.1 The simplicial complexes $K(\mathcal{U})$ are precisely those for which $\mathbb{Q}[K]$ is a complete intersection.

For the rest of this section we insist that $X$ is a 1 -connected space realising the complete intersection $\mathbb{Q}[K(\mathcal{U})]$, and maintain our convention of denoting the homotopy fibre of $q_{X}: X \rightarrow B T^{V}$ by $F_{X}$. In particular, the cofibre of any $q_{\mathrm{hc}(\partial(U))}$ is the Thom complex $\operatorname{Th}(U)$ of the universal product of line bundles over $B T^{U}$, so $q_{\mathrm{hc}(\partial(U))}$ is equivalent to the projection of the corresponding sphere bundle, and has homotopy fibre the $(2|U|-1)$-dimensional sphere $S(U)$; hence $F_{\mathrm{hc}(K(\mathcal{U}))}$ is given by $S(\mathcal{U}):=$ $S\left(U_{1}\right) \times \cdots \times S\left(U_{t}\right)$.

Lemma 9.2 The $p$-adic completion, rationalisation, and finite adele type of the fibration $F_{X} \rightarrow X \rightarrow B T^{V}$ are all themselves fibrations; moreover, $F_{X}$ is 1-connected and rationally equivalent to $S(\mathcal{U})$.

Proof The first statement follows from [2, Section II.5] since $B T^{V}$ is 1-connected; the 1-connectedness of $F_{X}$ arises directly from the definitions. Part I of [11, Proposition 5.11] confirms the existence of a homotopy equivalence $h: \operatorname{hc}(K(\mathcal{U}))_{0} \rightarrow X_{0}$, for which $q_{X} \circ h \simeq q_{\mathrm{hc}}(K(\mathcal{U})$ ) as rationalised maps. So $h$ lifts to a rational equivalence $S(\mathcal{U}) \rightarrow F_{X}$ of homotopy fibres. 
Now let $q^{\sharp}: X^{\sharp} \rightarrow B T^{V}$ denote the homotopy pullback of the fibration $q_{X}^{\wedge}$ along the completion map $B T^{V} \rightarrow\left(B T^{V}\right)^{\wedge}$; then the inclusion $F_{X}^{\wedge} \rightarrow X^{\sharp}$ of the fibre is homotopic to the principal $T^{V}$-fibration classified by $q^{\sharp}$.

Lemma 9.3 The homotopy fibre $F^{\sharp}$ of the induced map $X \rightarrow X^{\sharp}$ is a product $\prod_{i=1}^{t} H\left(\mathbb{Z}^{\wedge} / \mathbb{Z}, 2\left|U_{i}\right|-2\right)$ of Eilenberg-Mac Lane spaces.

Proof By construction, there is a homotopy pullback diagram

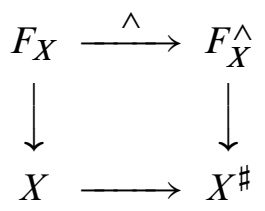

in which the homotopy fibre of the horizontal maps agrees with that of $\left(F_{X}\right)_{0} \rightarrow$ $\left(F_{X}\right)_{\mathbb{A}_{f}}$. Since the fibre of $S(\mathcal{U})_{0} \rightarrow S(\mathcal{U})_{\mathbb{A}_{f}}$ has the required form, the result follows from (40) and Lemma 9.2.

Theorem 9.4 Let $X$ be a nilpotent $C W$-complex, and suppose that $\mathbb{Q}[K]$ is a complete intersection; then there is an isomorphism $\theta: H^{*}(X ; \mathbb{Z}) \rightarrow \mathbb{Z}[K]$ if and only if there is a homotopy equivalence $f: \operatorname{hc}(K) \rightarrow X$ such that $f^{*}=\theta$.

Proof Using Proposition 9.1, write $K$ as $K(\mathcal{U})$ for some partition $\mathcal{U}$ of $V$. Then $\theta$ extends to an isomorphism $H^{*}\left(X ; \mathbb{Z}_{p}^{\wedge}\right) \rightarrow \mathbb{Z}_{p}^{\wedge}[K(\mathcal{U})]$ for each prime $p$, which is induced by an equivalence $\operatorname{hc}(K(\mathcal{U}))_{p}^{\wedge} \rightarrow X_{p}^{\wedge}$ via Theorem 8.6. So there exists a map $f^{\prime}: \operatorname{hc}(K(\mathcal{U})) \rightarrow X^{\wedge}$, such that $q_{X}^{\wedge} \circ f^{\prime}$ factors through the completion $B T^{V} \rightarrow$ $\left(B T^{V}\right)^{\wedge}$ using arguments of Sections 7 and 8 . In other words, the square

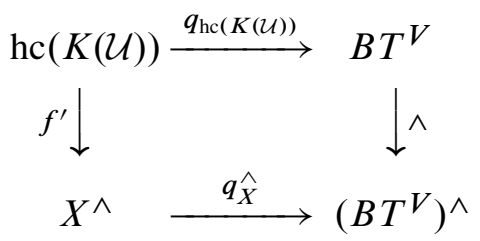

is homotopy commutative, and $f^{\prime}$ factors through $f^{\prime \prime}: \operatorname{hc}(K(\mathcal{U})) \rightarrow X^{\sharp}$.

To lift $f^{\prime \prime}$ to $X$, note that the obstructions lie in the groups $H^{i+1}\left(\operatorname{hc}(K(\mathcal{U})) ; \pi_{i}\left(F^{\sharp}\right)\right)$. But $\pi_{i}\left(F^{\sharp}\right)=0$ in odd dimensions by Lemma 9.3; so all obstructions vanish, and a lift $f: \operatorname{hc}(K(\mathcal{U})) \rightarrow X$ exists. Then $f^{*}=\theta$ by construction, and $f$ is an equivalence by Whitehead's theorem, because the source and target are 1-connected.

Theorem 9.5 Let $X$ be a $C W$-complex that realises $\mathbb{Z}[K]$ for any $K$; then there is a homotopy equivalence $e: \Sigma X \rightarrow \Sigma$ hc $(K)$. 
Proof The Stanley-Reisner algebra $\mathbb{Z}[K]$ decomposes additively as a sum $\oplus_{\sigma \in K} A_{\sigma}$ of graded subgroups, generated by monomials whose support is a particular $\sigma$. Every $A_{\sigma}$ is realised in $H^{*}(X ; \mathbb{Z})$ by a map $q_{\sigma}^{\prime}: X \rightarrow \wedge_{\sigma} B T$, defined by composing $q_{\sigma}$ with projection onto the iterated smash product. Using the cogroup structure of $\Sigma X$, we sum the suspensions $\Sigma q_{\sigma}^{\prime}$ over all faces $\sigma$, to obtain a map

$$
h_{X}: \Sigma X \longrightarrow \bigvee_{\sigma \in K} \Sigma\left(\wedge_{\sigma} B T\right)
$$

This induces a cohomology isomorphism of 1-connected CW-complexes, and is therefore a homotopy equivalence. Identical reasoning applies to $\mathrm{hc}(K)$, so it suffices to define $h$ as $h_{c(K)}^{-1} \circ h_{X}$.

We may use the splitting of [1, Theorem 1.18] as an elegant alternative to $h_{c(K)}$; in either event, Theorem 9.5 identifies $X$ with the wedge $\bigvee_{\sigma \in K} \operatorname{Th}(\sigma)$ of Thom complexes, after one suspension.

\section{References}

[1] A Bahri, M Bendersky, F R Cohen, S Gitler, The polyhedral product functor: a method of computation for moment-angle complexes, arrangements and related spaces arXiv:0711.4689

[2] A K Bousfield, D M Kan, Homotopy limits, completions and localizations, Lecture Notes in Math. 304, Springer, Berlin (1972) MR0365573

[3] W Bruns, J Herzog, Cohen-Macaulay rings, second edition, Cambridge Studies in Advanced Math. 39, Cambridge Univ. Press (1998) MR1251956

[4] V M Buchstaber, T E Panov, Torus actions and their applications in topology and combinatorics, Univ. Lecture Series 24, Amer. Math. Soc. (2002) MR1897064

[5] M W Davis, T Januszkiewicz, Convex polytopes, Coxeter orbifolds and torus actions, Duke Math. J. 62 (1991) 417-451 MR1104531

[6] F-X Dehon, J Lannes, Sur les espaces fonctionnels dont la source est le classifiant d'un groupe de Lie compact commutatif, Inst. Hautes Études Sci. Publ. Math. (1999) 127-177 (2000) MR1793415

[7] J Grbić, $\mathbf{S}$ Theriault, The homotopy type of the complement of a coordinate subspace arrangement, Topology 46 (2007) 357-396 MR2321037

[8] J Hollender, RM Vogt, Modules of topological spaces, applications to homotopy limits and $E_{\infty}$ structures, Arch. Math. (Basel) 59 (1992) 115-129 MR1170635

[9] N J Kuhn, M Winstead, On the torsion in the cohomology of certain mapping spaces, Topology 35 (1996) 875-881 MR1404914 
[10] J Lannes, Sur les espaces fonctionnels dont la source est le classifiant d'un p-groupe abélien élémentaire, Inst. Hautes Études Sci. Publ. Math. (1992) 135-244 MR1179079 With an appendix by M Zisman

[11] D Notbohm, N Ray, On Davis-Januszkiewicz homotopy types. I. Formality and rationalisation, Algebr. Geom. Topol. 5 (2005) 31-51 MR2135544

[12] B Oliver, Higher limits via Steinberg representations, Comm. Algebra 22 (1994) 13811393 MR1261265

[13] L Schwartz, Unstable modules over the Steenrod algebra and Sullivan's fixed point set conjecture, Chicago Lectures in Math., Univ. of Chicago Press (1994) MR1282727

[14] R P Stanley, Combinatorics and commutative algebra, second edition, Progress in Math. 41, Birkhäuser, Boston (1996) MR1453579

[15] R Thom, L'homologie des espaces fonctionnels, from: "Colloque de topologie algébrique, Louvain, 1956”, Georges Thone, Liège (1957) 29-39 MR0089408

[16] R M Vogt, Convenient categories of topological spaces for homotopy theory, Arch. Math. (Basel) 22 (1971) 545-555 MR0300277

[17] $\mathbf{Z}$ Wojtkowiak, On maps from ho $\lim F$ to $\mathbf{Z}$, from: "Algebraic topology, Barcelona, 1986", (J Aguadé, R Kane, editors), Lecture Notes in Math. 1298, Springer, Berlin (1987) 227-236 MR928836

[18] A Zabrodsky, On phantom maps and a theorem of H Miller, Israel J. Math. 58 (1987) 129-143 MR901174

Department of Mathematics, Vrije Universiteit Amsterdam

De Boelelaan 1081a, 1081 HV Amsterdam, The Netherlands

School of Mathematics, University of Manchester Oxford Road, Manchester M13 9PL, United Kingdom

notbohm@few.vu.nl, nige@ma.man.ac.uk

Received: 16 December $2008 \quad$ Revised: 8 April 2009 